\title{
CYCLIC OPERADS AND ALGEBRA OF CHORD DIAGRAMS
}

\author{
VLADIMIR HINICH AND ARKADY VAINTROB
}

\begin{abstract}
We prove that the algebra $\mathcal{A}$ of chord diagrams, the dual to the associated graded algebra of Vassiliev knot invariants, is isomorphic to the universal enveloping algebra of a Casimir Lie algebra in a certain tensor category (the PROP for Casimir Lie algebras). This puts on a firm ground a known statement that the algebra $\mathcal{A}$ "looks and behaves like a universal enveloping algebra". An immediate corollary of our result is the conjecture of [BGRT] on the Kirillov-Duflo isomorphism for algebras of chord diagrams.

Our main tool is a general construction of a functor from the category CycOp of cyclic operads to the category ModOp of modular operads which is left adjoint to the "tree part" functor ModOp $\rightarrow$ CycOp. The algebra of chord diagrams arises when this construction is applied to the operad LIE. Another example of this construction is Kontsevich's graph complex that corresponds to the operad $\operatorname{LIE}_{\infty}$ for homotopy Lie algebras.
\end{abstract}

\section{INTRODUCTION}

It is well known that the theory of knot invariants of finite type (or Vassiliev invariants) is closely connected with Lie algebras. The aim of this paper is to clarify this relationship and give it a precise formulation.

Knot invariants of finite type are related to various areas of mathematics and theoretical physics and have been in the focus of very intensive research ever since V. Vassiliev introduced them in 1989. One of the remarkable features of these invariants is that they can be completely characterized in terms of combinatorial objects called weight systems. A weight system is a function on chord diagrams (configurations of pairs of points on a circle) satisfying certain relations. The dual space $\mathcal{A}$ of the space of weight systems is generated by chord diagrams and has a natural structure of a graded commutative and cocommutative Hopf algebra. It is called the algebra of chord diagrams.

Bar-Natan [BN] and Kontsevich [K1] discovered a construction that gives a family of Vassiliev invariants for every finite-dimensional Lie 
algebra with a metric (an invariant inner product). According to calculations of J. Kneissler [Kn], all invariants up to order 12 come from Lie algebras. However for large orders this is not true, and there exists a more general construction that gives Vassiliev invariants which cannot be obtained from Lie algebras. This construction was found by the second author in $\mathbb{D}$ as a byproduct of an attempt to understand the relationship between Lie algebras and invariants of knots. It turned out that the theory of Vassiliev invariants naturally leads to the concept of a Yang-Baxter Lie algebra, an algebraic structure generalizing Lie algebras and Lie superalgebras, and they in turn can be used to produce knot invariants. Namely, every metric Yang-Baxter Lie algebra g gives an algebra homomorphism

$$
W_{\mathfrak{g}}: \mathcal{A} \rightarrow U(\mathfrak{g})
$$

with values in the center of the universal enveloping algebra of $\mathfrak{g}$. Every linear functional on $Z(U(\mathfrak{g}))$ produces a sequence of Vassiliev invariants.

The existence of homomorphism $W_{\mathfrak{g}}$ is not the only indication of the Lie-type behavior of the algebra $\mathcal{A}$ of chord diagrams. In particular, $\mathcal{A}$ can be described as the space generated by certain (ribbon) graphs (also known as Chinese characters) modulo some relations and the proof of this fact (see $[\mathrm{BN}]$ ) is strikingly parallel to the proof of the PoincaréBirkhoff-Witt theorem for Lie algebras. This raises a natural question whether the algebra $\mathcal{A}$ can be described as the center of the universal enveloping algebra of a Lie-type object which is universal with respect to morphisms (11).

In this paper we show that this is, indeed, the case and prove, in particular, that every Vassiliev invariant factors through the homomorphism $W_{\mathfrak{g}}$ for some $\mathfrak{g}$.

This universal object, however, cannot be found among Yang-Baxter Lie algebras, and to define it we need to move to a slightly higher level of abstraction.

Metric Lie algebras can be defined not only in the category of vector spaces, but in arbitrary linear tensor category. One can construct a metric Lie algebra $\mathbb{L}^{M}$ in a certain category $\operatorname{LIE}^{M}$ universal in the sense that every metric Lie algebra in a tensor category $\mathcal{C}$ is the image of $\mathbb{L}^{M}$ under a unique tensor functor $\operatorname{LIE}^{M} \rightarrow \mathcal{C}$.

A Lie algebra $\mathfrak{g}$ in a tensor category has a universal enveloping algebra $U(\mathfrak{g})$ which is an ordinary associative algebra in the category of vector spaces. The collection of maps (1) can now be described as 
an algebra homomorphism $\mathcal{A} \rightarrow U\left(\mathbb{L}^{M}\right)$. This homomorphism however is not an isomorphism, and the starting point of our work was to understand to which extent it determines the algebra $\mathcal{A}$.

An appropriate setup is provided by the more general notion of a Casimir Lie algebra (i.e. Lie algebra with an invariant symmetric twotensor). Similarly to the case of metric Lie algebras, we construct the universal Casimir Lie algebra $\mathbb{L}^{C}$ in a certain tensor category $\operatorname{LIE}^{C}$ and a homomorphism $U\left(\mathbb{L}^{C}\right) \rightarrow \mathcal{A}$. One of the main results of the paper is that this map is an isomorphism.

This result can be derived from injectivity of the natural map $U\left(\mathbb{L}^{C}\right) \rightarrow$ $U\left(\mathbb{L}^{M}\right)$ which, in turn, follows from a detailed analysis of the categories $\mathrm{LIE}^{C}$ and $\operatorname{LIE}^{M}$.

To present the main constructions and results of the paper we need to use the language of operads and PROPs.

The notion of operad appeared in algebraic topology in late 60s as a tool for describing algebraic operations on iterated loop spaces. In algebra, operads are used to encode classes of algebraic structures (algebras over operads) with collections of polylinear operations of type $L^{\otimes n} \rightarrow L$ satisfying specific properties. In particular, there exists an operad called LIE, such that LIE-algebras in different tensor categories include "usual" Lie algebras, Lie superalgebras, as well as dg-Lie algebras and Yang-Baxter Lie algebras. For every operad $\mathcal{O}$ there exists a free $\mathcal{O}$-algebra with a given set of generators.

An invariant inner product on an algebra $L$ over a field $k$ can be considered as an operation $b: L^{\otimes 2} \rightarrow k=L^{\otimes 0}$. The notions of PROP and algebras over PROPs have been designed to handle this and more general operations of type $L^{\otimes n} \rightarrow L^{\otimes m}$.

As in the case of operads, the formalism of PROPs allows to define algebras in arbitrary tensor categories. PROP itself is a very small tensor category: its objects are natural numbers $\mathbf{n} \in \mathbb{N}=\{\mathbf{0}, \mathbf{1}, \mathbf{2}, \ldots\}$ with the tensor structure given by addition. Mentioned above categories $\mathrm{LIE}^{M}$ and $\mathrm{LIE}^{C}$ are, in fact, PROPs, such that the corresponding algebras are exactly metric and Casimir Lie algebras. In particular, the objects

$$
\mathbb{L}^{M}=\mathbf{1} \in \mathrm{LIE}^{M} \quad \text { and } \quad \mathbb{L}^{C}=\mathbf{1} \in \mathrm{LIE}^{C}
$$

are Lie algebras in the corresponding categories and they can be viewed as the universal metric Lie algebra and the universal Casimir Lie algebra respectively.

Similarly to the definition of an enveloping algebra of an algebra over an operad (see [HS, Sect. 3), one can consider two different versions of a universal enveloping algebra of the Lie algebra $\mathbb{L}^{M} \in \operatorname{LIE}^{M}$ (resp. 
$\left.\mathbb{L}^{C} \in \mathrm{LIE}^{C}\right)$. The first, internal universal enveloping algebra, is an associative algebra in a certain extension of the category $\mathrm{LIE}^{C}$ (resp. $\mathrm{LIE}^{M}$ ). The second, external universal enveloping algebra, is a genuine associative algebra. This algebra can be described as a collection of compatible endomorphisms of all representations of $\mathbb{L}^{M}$ (resp. $\mathbb{L}^{C}$ ).

The homomorphisms (1) giving Vassiliev invariants for arbitrary metric Yang-Baxter Lie algebras now can be interpreted as a single algebra homomorphism

$$
W_{\mathbb{L}^{M}}: \mathcal{A} \rightarrow U^{M}
$$

from the algebra of chord diagrams to the external enveloping algebra of the universal metric Lie algebra $\mathbb{L}^{M} \in \mathrm{LIE}^{M}$.

A precise Lie-theoretical description of the algebra of chord diagrams is obtained when we replace metric Lie algebras by Casimir Lie algebras. The following theorem is the central result of the paper.

Theorem. There exists an algebra isomorphism

$$
U^{C} \stackrel{\sim}{\longrightarrow} \mathcal{A}
$$

from the external enveloping algebra of the universal Casimir Lie algebra $\mathbb{L}^{C} \in \mathrm{LIE}^{C}$ to the algebra $\mathcal{A}$ of chord diagrams.

As an immediate consequence, we see that each Casimir Lie algebra $\mathfrak{g}$ gives rise to a homomorphism from $\mathcal{A}$ to the center of the enveloping algebra of $\mathfrak{g}$ and that every Vassiliev invariant can be obtained from a Casimir Lie algebra in some tensor category.

This explains the similarities between the algebra of chord diagrams and Lie algebras. In particular, the above-mentioned description of the algebra $\mathcal{A}$ in terms of Chinese characters follows from the PoincaréBirkhoff-Witt theorem for $U^{C}$. Another immediate corollary of this theorem is the conjecture of Bar-Natan, Garoufalidis, Rozansky, and Thurston BGRT on the existence of a Kirillov-Duflo-type isomorphism for algebras of chord diagrams.

A large part of our results and constructions for metric and Casimir Lie algebras remains true if the operad LIE is replaced by an arbitrary cyclic operad $\mathcal{O}$. In particular, we construct a PROP $\mathcal{O}^{C}$ describing $\mathcal{O}$-algebras endowed with an invariant symmetric two-tensor. It turns out that the operadic part $\mathbb{M}(\mathcal{O})$ of $\mathcal{O}^{C}$ has an extra structure that of a modular operad. Moreover, the functor $\mathcal{O} \mapsto \mathbb{M}(\mathcal{O})$ is a left adjoint to the natural "tree part" functor from modular operads to cyclic operads. We give an explicit construction of $\mathbb{M}(\mathcal{O})$ in terms of $\mathcal{O}$. The PROP $\mathcal{O}^{C}$ can be expressed through $\mathbb{M}(\mathcal{O})$ in a simple way. Similarly, the PROP $\mathcal{O}^{M}$ also can be described in terms of $\mathbb{M}(\mathcal{O})$. The 
explicit description of PROPs $\mathcal{O}^{C}$ and $\mathcal{O}^{M}$ allows one to deduce the following result which is the key ingredient in our characterization of the algebra $\mathcal{A}$.

Theorem. The natural morphism of PROPs $\mathcal{O}^{C} \rightarrow \mathcal{O}^{M}$ induces an isomorphism

$$
\operatorname{Hom}_{\mathcal{O}^{M}}(\mathbf{0}, \mathbf{0}) \otimes \operatorname{Hom}_{\mathcal{O}^{C}}(\mathbf{0}, \mathbf{n}) \stackrel{\sim}{\longrightarrow} \operatorname{Hom}_{\mathcal{O}^{M}}(\mathbf{0}, \mathbf{n}) .
$$

When $\mathcal{O}=$ LIE this theorem implies in particular that all Vassiliev invariants can be obtained from a metric Lie algebra.

Another interesting example is the case when $\mathcal{O}=\mathrm{LIE}_{\infty}$, the operad for homotopy Lie algebras. In this case the space of morphisms in $\operatorname{LIE}_{\infty}^{C}$ coincides with Kontsevich's graph complex.

The paper is organized as follows. The constructions and results of the first part (Sections $2-4$ ) are valid for arbitrary cyclic operads. We believe that they may find other applications besides the ones we discuss in the second part of the paper (Sections $5-7$ ).

Section 2 describes our conventions about tensor categories, operads, and PROPs. In Section 3 we study cyclic and modular operads and algebras over them. We construct various functors between the categories of cyclic and modular operads and PROPs. In Section 4 we prove some results on adjointness and isomorphisms for these functors. In Section 5 we discuss various versions of the notion of universal enveloping algebra for algebras over operads and PROPs. We prove that when $\mathcal{O}=$ LIE internal enveloping algebras exist in a certain extension of the tensor category $\mathcal{P}$ and then study them in detail in the cases when $\mathcal{P}=\operatorname{LIE}^{M}$ and $\mathcal{P}=\mathrm{LIE}^{C}$. In Section 6 we review basic facts about Vassiliev knot invariants and their relations with Lie algebras. Finally, in Section 7, we present several applications of the results of the previous sections. In particular, we show how results of Section 5 allow to describe the algebra $\mathcal{A}$ of chord diagrams as the external universal enveloping algebra of the universal Casimir Lie algebra $\mathbb{L}^{C}$.

Acknowledgments. This work was started when both authors were visiting Max-Planck-Institut für Mathematik in Bonn and finished when we both were at MSRI in Berkeley. We express our gratitude to these Institutes for hospitality and financial support.

Also we would like to thank Silvio Levy for showing us how to make $\mathbb{1 1}$. 


\section{PRELIMINARIES}

\subsection{Tensor categories.}

By a tensor category we understand a $k$-linear symmetric monoidal category (see [DM, D]) over a field $k$ of characteristic zero. The unit object in a tensor category will be usually denoted by $\mathbb{1}$. For any object $A$ in a tensor category, the associativity constraint allows to define uniquely (up to a unique isomorphism) the tensor powers $A^{\otimes n}$ and the commutativity constraint gives a left action of the symmetric group $\Sigma_{n}$ on $A^{\otimes n}$.

Recall the following definition.

2.1.1. Definition. An object $A$ of a tensor category $\mathcal{C}$ is called rigid if there exists an object $A^{\vee} \in \mathcal{C}$ and a pair of morphisms

$$
\phi: \mathbb{I} \rightarrow A^{\vee} \otimes A, \quad \psi: A \otimes A^{\vee} \rightarrow \mathbb{I},
$$

such that the compositions

$$
A^{\vee} \stackrel{\phi \otimes \mathrm{id}}{\longrightarrow} A^{\vee} \otimes A \otimes A^{\vee} \stackrel{\mathrm{id} \otimes \psi}{\longrightarrow} A^{\vee}
$$

and

$$
A \stackrel{\operatorname{id} \otimes \phi}{\longrightarrow} A \otimes A^{\vee} \otimes A \stackrel{\psi \otimes \mathrm{id}}{\longrightarrow} A
$$

are the identities.

The object $A^{\vee}$ is called the dual of $A$. The dual object, if it exists, is unique up to a unique isomorphism. The pair $(\phi, \psi)$ is called an adjoint pair. Given one of the morphisms $\phi$ or $\psi$, its adjoint, if it exists, is unique.

2.1.2. Example. Let $\mathcal{C}$ be the category Vect of $k$-vector spaces. Then $V \in \mathcal{C}$ is rigid if and only if $\operatorname{dim} V<\infty$.

\subsection{PROPs and algebras over them.}

Here we recall some basic facts about PROPs, operads, and algebras over them. Standard references for this material are [BV, M, May, A]. See also EK, 1.1, 1.2.

2.2.1. Denote by $\mathbb{S}$ the tensor category whose objects are non-negative integers $\mathbf{0}, \mathbf{1}, \mathbf{2}, \ldots$, and morphisms are given by

$$
\operatorname{Hom}_{\mathbb{S}}(\mathbf{m}, \mathbf{n})= \begin{cases}\emptyset, & m \neq n \\ \Sigma_{n}, & m=n,\end{cases}
$$

where $\Sigma_{n}$ is the symmetric group on $n$ objects. Tensor product in $\mathbb{S}$ is given by the addition of numbers; commutativity constraint

$$
s_{m n}: \mathbf{m} \otimes \mathbf{n} \rightarrow \mathbf{n} \otimes \mathbf{m}
$$


is defined by the shuffle

$$
s_{m n} \in \Sigma_{m+n}, \quad s_{m n}(i)=\left\{\begin{array}{ll}
i+n, & i \leq m \\
i-m, & i>m
\end{array} .\right.
$$

The category $\mathbb{S}$ is the simplest example of a $\operatorname{PROP}($ see $[\mathbb{M}]$ ).

2.2.2. Definition. A PROP is a tensor category $\mathcal{P}$ with $\mathrm{Ob} \mathcal{P}=$ $\{\mathbf{0}, \mathbf{1}, \mathbf{2}, \ldots\}$ and a tensor functor $\mathbb{S} \rightarrow \mathcal{P}$ identical on objects and injective on morphisms.

For a $\operatorname{PROP} \mathcal{P}$, we will write $\mathcal{P}(m, n)$ instead of $\operatorname{Hom}_{\mathcal{P}}(\mathbf{m}, \mathbf{n})$.

A morphism of PROPs $f: \mathcal{P} \rightarrow \mathcal{P}^{\prime}$ is a functor from $\mathcal{P}$ to $\mathcal{P}^{\prime}$ which is identical on $\mathbb{S}$. The category of PROPs will be denoted by Props.

2.2.3. Definition. Let $\mathcal{P}$ be a $\mathrm{PROP}$ and let $\mathcal{C}$ be a tensor category. A $\mathcal{P}$-algebra in $\mathcal{C}$ is a tensor functor $A: \mathcal{P} \rightarrow \mathcal{C}$.

2.2.4. Example. Let Vect be the category of $k$-vector spaces. A $\mathcal{P}$ algebra in Vect is a vector space $V=A(\mathbf{1})$ together with a compatible collection of operations $A(p): V^{\otimes m} \rightarrow V^{\otimes n}$, for each $p \in \mathcal{P}(m, n)$.

2.2.5. Operads. By an operad in this paper we understand a collection of vector spaces $\mathcal{O}=\{\mathcal{O}(n)\}, n \in \mathbb{N}$, endowed with a right action of the symmetric group $\Sigma_{n}$ on $\mathcal{O}(n)$ and a collection of composition maps

$$
\gamma: \mathcal{O}(n) \otimes \mathcal{O}\left(m_{1}\right) \otimes \ldots \otimes \mathcal{O}\left(m_{n}\right) \rightarrow \mathcal{O}\left(\sum m_{i}\right)
$$

satisfying natural conditions of equivariance, associativity, and unity (see May, HS).

An algebra over an operad $\mathcal{O}$ is a vector space $A$ with a collection of operations

$$
\mathcal{O}(n) \otimes A^{\otimes n} \rightarrow A
$$

satisfying natural compatibility conditions.

The category of operads will be denoted by $0 \mathrm{p}$.

2.2.6. PROPs and operads. There exists a pair of adjoint functors

$$
\text { \# : Props } \rightarrow \text { Op and } \mathrm{P}: \mathrm{Op} \rightarrow \text { Props, }
$$

where $\mathcal{P}^{\#}(n)=\mathcal{P}(n, 1)$, and the left adjoint to \# functor $\mathrm{P}$ is defined by the formula

$$
\mathrm{P}(\mathcal{O})(m, n)=\bigoplus_{f} \bigotimes_{i=1}^{n} \mathcal{O}\left(\left|f^{-1}(i)\right|\right),
$$

where $\mathcal{O}$ is an operad and the direct sum is taken over all maps

$$
f:\{1, \ldots, m\} \rightarrow\{1, \ldots, n\} .
$$


The notions of algebras over PROPs and over operads are compatible: an algebra over an operad $\mathcal{O}$ is the same as an algebra over the PROP $\mathrm{P}(\mathcal{O})$.

By a map of an operad $\mathcal{O}$ to a PROP $\mathcal{P}$ we understand a morphism of operads $\mathcal{O} \rightarrow \mathcal{P}^{\#}$.

\section{CYCLIC AND MODUlaR OPERADS}

3.1. Metric and Casimir algebras. In this section we define two types of algebras over a cyclic operad. One type, that of metric algebras, is well known. The other one generalizes the concept of Lie algebra endowed with a casimir element.

These two types of algebras are governed by two different PROPs which will be the main objects of study in the paper.

3.1.1. Cyclic operads. A cyclic operad (see GKQ) is an operad $\mathcal{O}$ with a right action of the symmetric group $\Sigma_{n+1}$ on $\mathcal{O}(n)$ extending the $\Sigma_{n}$ action and satisfying the compatibility condition (5) below.

Note that the symmetric group $\Sigma_{n+1}$ is generated by the subgroup $\Sigma_{n}$ (identified with the stabilizer of $0 \in\{0, \ldots, n\}$ ) and by the cyclic permutation $\tau$ given by $\tau(i)=i-1$ for $i>0$ and $\tau(0)=n$.

The operad structure on $\mathcal{O}$ can be described in terms of composition operations

$$
\mathrm{o}_{i}: \mathcal{O}(m) \otimes \mathcal{O}(n) \rightarrow \mathcal{O}(m+n-1), i=1, \ldots, m,
$$

corresponding to the insertion of an element of $\mathcal{O}(n)$ as the $i$-th argument of an element of $\mathcal{O}(m)$.

Definition. An operad $\mathcal{O}$ with a collection of right $\Sigma_{n+1}$-actions on $\mathcal{O}(n)$ is called cyclic if

$$
\left(a \circ_{1} b\right) \tau=(b \tau) \circ_{n}(a \tau) \text { for } a \in \mathcal{O}(m), b \in \mathcal{O}(n) .
$$

The category of cyclic operads will be denoted by CycOp.

3.1.2. Definition. Let $\mathcal{O}$ be a cyclic operad. A metric $\mathcal{O}$-algebra in a tensor category $\mathcal{C}$ is an $\mathcal{O}$-algebra $A \in \mathcal{C}$ together with a symmetric form $b: A \otimes A \rightarrow \mathbb{I}$ satisfying the following conditions.

(i) The form $b$ is non-degenerate, that is there exists a two-tensor $c: \mathbb{I} \rightarrow A \otimes A$ adjoint to $b$ in the sense of Definition 2.1.1.

(ii) The form $b$ is $\mathcal{O}$-invariant, that is the composition

$$
\mathcal{O}(n) \otimes A^{\otimes n+1} \rightarrow A \otimes A \stackrel{b}{\longrightarrow} \mathbb{I}
$$

is $\Sigma_{n+1}$-invariant.

In GKC metric algebras are called cyclic algebras. 
3.1.3. PROP for metric algebras. The notion of a metric algebra gives rise to the following construction.

For a cyclic operad $\mathcal{O}$ let $\mathcal{O}^{M}$ be the PROP generated by the PROP $\mathrm{P}(\mathcal{O})$ (see (身) ) and two elements $b \in \mathcal{O}^{M}(2,0)$ and $c \in \mathcal{O}^{M}(0,2)$ satisfying the following conditions.

(i) The morphisms $b$ and $c$ are symmetric and mutually adjoint.

(ii) (invariance) For each $f \in \mathcal{O}(n)$ the composition

$$
\mathbf{n} \stackrel{c \otimes i d}{\longrightarrow} \mathbf{2} \otimes \mathbf{n}=\mathbf{1} \otimes \mathbf{n} \otimes \mathbf{1} \stackrel{\mathrm{id} \otimes f \otimes \mathrm{id}}{\longrightarrow} \mathbf{1} \otimes \mathbf{1} \otimes \mathbf{1} \stackrel{\mathrm{id} \otimes b}{\longrightarrow} \mathbf{1}
$$

is equal to $f \tau$.

The following simple result explains the meaning of $\mathcal{O}^{M}$.

3.1.4. Lemma. Metric $\mathcal{O}$-algebras are precisely the algebras over the $\operatorname{PROP} \mathcal{O}^{M}$.

For a cyclic operad $\mathcal{O}$ there exists a canonical morphism

$$
i_{M}^{\mathcal{O}}: \mathcal{O} \rightarrow \mathcal{O}^{M}
$$

to the corresponding PROP. This morphism gives the functor

$$
i_{M *}^{\mathcal{O}}: \operatorname{Alg}\left(\mathcal{O}^{M}\right) \rightarrow \operatorname{Alg}(\mathcal{O})
$$

that forgets the metric of a metric $\mathcal{O}$-algebra.

3.1.5. Definition. Let $\mathcal{O}$ be a cyclic operad. A Casimir $\mathcal{O}$-algebra in a tensor category $\mathcal{C}$ is an $\mathcal{O}$-algebra $A \in \mathcal{C}$ together with a symmetric $\mathcal{O}$-invariant two-tensor (called casimir) $c: \mathbb{I} \rightarrow A \otimes A$.

The condition of $\mathcal{O}$-invariance means that the following composition

$$
\mathcal{O}(n)=\mathcal{O}(n) \otimes \mathbb{I}^{\otimes n} \stackrel{c^{\otimes n}}{\longrightarrow} \mathcal{O}(n) \otimes\left(A^{\otimes 2}\right)^{\otimes n} \rightarrow A \otimes A^{\otimes n}=A^{\otimes n+1}
$$

is $\Sigma_{n+1}$-equivariant with respect to the standard $\Sigma_{n+1}$-action on $A^{\otimes n+1}$ given by

$$
x \sigma=\sigma^{-1}(x) \text { for } x \in A^{\otimes n+1} \text {, and } \sigma \in \Sigma_{n+1} .
$$

3.1.6. PROP for Casimir algebras. Similarly to 3.1.3 we construct a PROP responsible for Casimir algebras.

Let $\mathcal{O}$ be a cyclic operad. Denote by $\mathcal{O}^{C}$ the PROP generated by the PROP $\mathrm{P}(\mathcal{O})$ (see 2.2.6) and a symmetric element $c \in \mathcal{O}^{C}(0,2)$ satisfying the following invariance condition. 
For each $f \in \mathcal{O}(n)$ the diagram

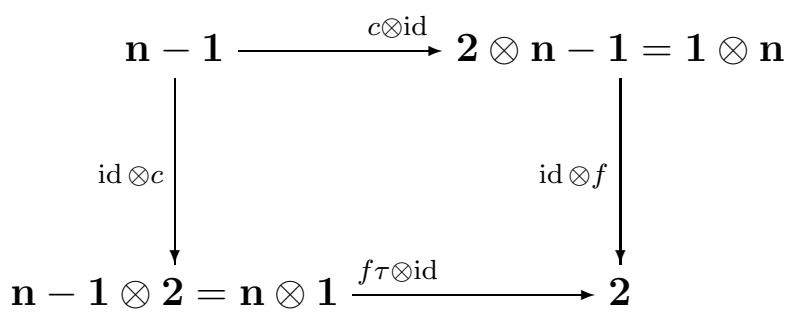

is commutative.

The following fact is an analog of Lemma 3.1 .4 for Casimir algebras.

3.1.7. Lemma. Casimir $\mathcal{O}$-algebras are precisely the algebras over the PROP $\mathcal{O}^{C}$.

For a cyclic operad $\mathcal{O}$ there is a canonical morphism

$$
i_{C}^{\mathcal{O}}: \mathcal{O} \rightarrow \mathcal{O}^{C}
$$

to the corresponding PROP for Casimir $\mathcal{O}$-algebras. This morphism gives the functor

$$
i_{C *}^{\mathcal{O}}: \operatorname{Alg}\left(\mathcal{O}^{C}\right) \rightarrow \operatorname{Alg}(\mathcal{O})
$$

that forgets the casimir of a Casimir $\mathcal{O}$-algebra.

It is easy to see that algebras with invertible casimirs are metric algebras.

3.1.8. Lemma. Let $A$ be an algebra over a cyclic operad $\mathcal{O}$ and

$$
b: A \otimes A \rightarrow \mathbb{I} \text { and } c: \mathbb{I} \rightarrow A \otimes A
$$

be a pair of symmetric mutually adjoint maps. Then $b$ satisfies the conditions (i), (ii) of Definition 3.1.9 if and only if $c$ is $\mathcal{O}$-invariant in the sense of Definition 3.1.5.

This lemma gives a functor

$$
\operatorname{Alg}\left(\mathcal{O}^{M}\right) \rightarrow \operatorname{Alg}\left(\mathcal{O}^{C}\right)
$$

that commutes with $i_{M *}^{\mathcal{O}}$ and $i_{C *}^{\mathcal{O}}$ and is induced by a morphism of PROPs

$$
\mathcal{O}^{C} \rightarrow \mathcal{O}^{M}
$$

commuting with $i_{M}^{\mathcal{O}}$ and $i_{C}^{\mathcal{O}}$.

The goal of this section is to give a detailed description of the relationship between the PROPs $\mathcal{O}^{M}$ and $\mathcal{O}^{C}$. 


\subsection{Coordinate-free language.}

In this paper, when dealing with tensor categories and operads we will use a "coordinate-free" language of [DM. It allows one to hide some "ugly" part of structure (the associativity and commutativity constraints, action of symmetric group, etc.) inside the category of finite sets. In this subsection we recall the basic definitions and reformulate the notion of a cyclic operad in the new language. In the following subsection we will use this language to describe modular operads.

The following is a coordinate-free definition of tensor category (see [DM, Prop. 1.5).

3.2.1. Definition. A tensor category $\mathcal{C}$ is a category with functors

$$
\bigotimes_{I}: \mathcal{C}^{I} \rightarrow \mathcal{C}:\left(X_{i}, i \in I\right) \mapsto \bigotimes_{i \in I} X_{i}
$$

and functorial isomorphisms

$$
\chi(\alpha): \bigotimes_{i \in I} X_{i} \stackrel{\sim}{\longrightarrow} \bigotimes_{j \in J}\left(\bigotimes_{i \in \alpha^{-1}(j)} X_{i}\right)
$$

defined for each finite set $I$ and each map $\alpha: I \rightarrow J$ of finite sets. The functors $\bigotimes_{I}$ and isomorphisms $\chi(\alpha)$ have to satisfy the following conditions.

(i) If $I$ consists of a single element, then $\otimes_{i \in I}$ is the identity functor, and for any map $\alpha$ between one-element sets $\chi(\alpha)$ is the identity automorphism of the identity functor.

(ii) The functors $\chi(\alpha)$ satisfy a natural associativity condition relating $\chi(\beta \circ \alpha)$ with $\chi(\beta)$ and $\chi(\alpha)$ for any pair of maps $I \stackrel{\alpha}{\longrightarrow} J \stackrel{\beta}{\longrightarrow} K$ of finite sets.

3.2.2. PROPs in the coordinate-free language. In the new language the initial PROP $\mathbb{S}$ (see 2.2.1) is replaced by the groupoid of finite sets with tensor product given by the operaton of disjoint union. As a tensor category it is equivalent to the category of $\mathbb{S}$ of 2.2 .1 and we will denote it by the same symbol.

A PROP in this setting is defined as a tensor category $\mathcal{P}$ with a tensor functor from $\mathbb{S}$ to $\mathcal{P}$ bijective on objects and injective on morphisms.

3.2.3. Cyclic operads in the coordinate-free language. Denote by $\mathbb{S}^{*}$ the groupoid of non-empty finite sets. A cyclic operad is a functor

$$
\mathcal{O}: \mathbb{S}^{*} \rightarrow \text { Vect }
$$


with a collection of functorial composition operations

$$
\circ_{x y}: \mathcal{O}(X) \otimes \mathcal{O}(Y) \rightarrow \mathcal{O}(X \sqcup Y \backslash\{x, y\})
$$

defined for each pair of pointed sets $(X, x)$ and $(Y, y)$ satisfying the following conditions.

(i) (commutativity) Operations $\circ_{x y}$ and $\circ_{y x}$ coincide after canonical identification of $X \sqcup Y$ with $Y \sqcup X$.

(ii) (associativity) For $x \in X, \quad y, y^{\prime} \in Y, y \neq y^{\prime}, \quad z \in Z$, the following diagram is commutative

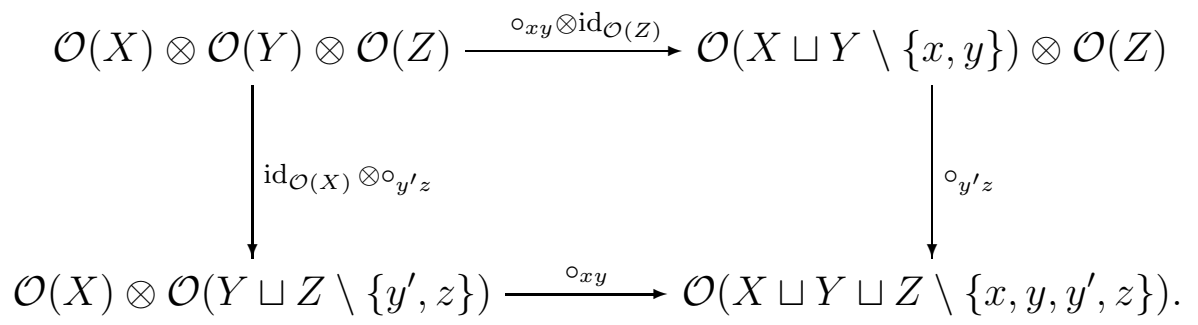

(iii) (unity) For every two-element set $\{p, q\}$, there is a distinguished element $I_{p q} \in \mathcal{O}(\{p, q\})$, such that for any $x \in X$ and $a \in \mathcal{O}(X)$, the elements $a$ and $a o_{x y} I_{y z}$ coincide after identifying $X$ with $X \cup\{z\} \backslash\{x\}$.

The space $\mathcal{O}(X)$ should be viewed as a set of "relations" with arguments labelled by $X$. The composition $\circ_{x y}$ corresponds to the operation of gluing the sets $X$ and $Y$ along the points $x$ and $y$.

\subsection{Modular operads.}

Roughly speaking, modular operads are cyclic operads where gluing operations similar to (13) are allowed for arbitrary non-empty subsets $U \subset X$ and $V \subset Y$. We will use two different kinds of modular operads (see definitions 3.3.1 and 3.5.3).

Modular operads were introduced by Getzler and Kapranov in GKM. Our definitions differ slightly from the one given in GKM - see 3.5.6 for a comparison.

3.3.1. Definition. A modular operad is a collection of functors

$$
\mathcal{M}^{n}: \mathbb{S}^{*} \rightarrow \text { Vect }, \quad n=0,1, \ldots
$$

with composition operations

$$
\circ_{f}: \mathcal{M}^{m}(X) \otimes \mathcal{M}^{n}(Y) \rightarrow \mathcal{M}^{m+n+d-1}(X \sqcup Y \backslash(U \sqcup V))
$$


defined for each bijection $U \stackrel{f}{\longrightarrow} V$ between non-empty $d$-element subsets $U \subset X$ and $V \subset Y$ with

$$
X \sqcup Y \backslash(U \sqcup V) \neq \emptyset
$$

satisfying the following conditions.

(i) (commutativity) Operations $\circ_{f}$ and $\circ_{f^{-1}}$ coincide after the canonical identification of $X \sqcup Y$ with $Y \sqcup X$.

(ii) (associativity) For $i=1,2,3$, let $X_{i}$ be a non-empty finite set with two disjoint subsets $U_{i j} \subseteq X_{i}, j \in\{1,2,3\} \backslash\{i\}$. Let, in addition, $f_{i j}: U_{i j} \rightarrow U_{j i}$ be bijections satisfying $f_{i j}=f_{j i}^{-1}$.

If $U_{13}=U_{31}=\emptyset$ and $U_{12}$ and $U_{23}$ are non-empty, then

$$
\circ_{f_{23}}\left(\circ_{f_{12}} \otimes \mathrm{id}_{X_{3}}\right)=\circ_{f_{21}}\left(\circ_{f_{23}} \otimes \mathrm{id}_{X_{1}}\right) .
$$

If all the subsets $U_{i j}$ are non-empty, then the following three maps from $\bigotimes_{i=1}^{3} \mathcal{M}\left(X_{i}\right)$ to $\mathcal{M}\left(\bigsqcup_{i}\left(X_{i} \backslash \bigcup_{j} U_{i j}\right)\right)$ coincide:

$$
\circ_{f_{13} \sqcup f_{23}}\left(\circ_{f_{12}} \otimes \operatorname{id}_{X_{3}}\right)=\circ_{f_{12} \sqcup f_{32}}\left(\circ_{f_{13}} \otimes \operatorname{id}_{X_{2}}\right)=\circ_{f_{21} \sqcup f_{31}}\left(\circ_{f_{23}} \otimes \operatorname{id}_{X_{1}}\right) .
$$

(iii) (unity) See 3.2.3(iii).

The category of modular operads will be denoted by ModOp.

The following proposition gives an equivalent definition of a modular operad (see GKM, $3.4-3.7$ ).

3.3.2. Proposition. A modular operad is a graded cyclic operad

$$
\mathcal{M}=\bigoplus_{n \geq 0} \mathcal{M}^{n}: \mathbb{S}^{*} \rightarrow \text { Vect }
$$

endowed with contraction operations

$$
c_{x y}: \mathcal{M}^{n}(X) \rightarrow \mathcal{M}^{n+1}(X \backslash\{x, y\}), \quad x, y \in X, x \neq y, X \neq\{x, y\}
$$

satisfying the following properties.

(i) $c_{x y}=c_{y x}$.

(ii) If $x, y, z, t \in X$ are four distinct elements, then the contractions $c_{x y}$ and $c_{z t}$ commute.

(iii) Let $x_{1} \neq x_{2} \in X, \quad y_{1} \neq y_{2} \in Y$ and $X \sqcup Y \neq\left\{x_{1}, x_{2}, y_{1}, y_{2}\right\}$. Then the operations $c_{x_{1}, y_{1}} \circ_{x_{2}, y_{2}}$ and $c_{x_{2}, y_{2}} \circ_{x_{1}, y_{1}}$ from $\mathcal{M}(X) \otimes \mathcal{M}(Y)$ to $\mathcal{M}\left(X \sqcup Y \backslash\left\{x_{1}, x_{2}, y_{1}, y_{2}\right\}\right)$ coincide. 
Proof. Suppose $\mathcal{M}=\left\{\mathcal{M}^{n}: \mathbb{S}^{*} \rightarrow\right.$ Vect $\}$ is a modular operad in the sense of 3.3.1. Then one can define the contraction operation

$$
c_{x y}: \mathcal{M}^{n}(X) \rightarrow \mathcal{M}^{n+1}(X \backslash\{x, y\})
$$

as the composition with the identity $I_{x^{\prime} y^{\prime}} \in \mathcal{M}^{0}\left(\left\{x^{\prime}, y^{\prime}\right\}\right)$ under the map $f$ sending $x^{\prime}$ to $x$ and $y^{\prime}$ to $y$. Property (i) follows from 3.3.1(i), property (ii) follows from 3.3.1(ii) with $X_{2}=X, X_{1}=\{x, y\}$, and $X_{3}=\{z, t\}$. Property (iii) follows from the fact that both compositions coincide with $\circ_{f}$, where $f:\left\{x_{1}, x_{2}\right\} \longrightarrow\left\{y_{1}, y_{2}\right\}: f\left(x_{i}\right)=y_{i}$.

Consider now a graded cyclic operad $\mathcal{M}$ endowed with a collection of contractions satisfying properties (i)-(iii) above. Define compositions

$$
\circ_{f}: \mathcal{M}^{m}(X) \otimes \mathcal{M}^{n}(Y) \rightarrow \mathcal{M}^{m+n+d-1}(X \sqcup Y \backslash(U \sqcup V))
$$

as follows. Choose $u \in U$ and define $\circ_{f}$ to be the composition of $\circ_{u, f(u)}$ with the contractions $c_{v, f(v)}$ for all $v \in U \backslash\{u\}$. The result does not depend on the choice of $u \in U$ and on the order of the contractions by the properties (ii) and (iii) of contractions.

The following is a non-graded version of the notion of a modular operad.

3.3.3. Definition. A non-graded modular operad is a cyclic operad $\mathcal{M}$ together with contraction operations

$$
c_{x y}: \mathcal{M}(X) \rightarrow \mathcal{M}(X \backslash\{x, y\}), \quad x, y \in X, x \neq y, X \neq\{x, y\}
$$

satisfying properties (i)-(iii) of Proposition 3.3 .2

The category of non-graded modular operads will be denoted by ModOp ${ }^{n g r}$.

\subsection{Standard functors.}

Here we will construct several functors connecting various categories of operads and PROPs.

3.4.1. From modular operads to PROPs. We start with a natural construction that associates a PROP to a modular operad.

Proposition. There exists a functor

$$
\mathbb{P}: \text { ModOp } \rightarrow \text { Props }
$$

with $\mathbb{P}(\mathcal{M})(X, Y)$ given for $X, Y \in \mathbb{S}$ by the formula

$$
\mathbb{P}(\mathcal{M})(X, Y)=\bigoplus_{\substack{X=\coprod_{i \in I} X_{i} \\ Y=\coprod_{i \in I} Y_{i} \\ Y_{i} \neq \emptyset}} \bigotimes_{i \in I} \mathcal{M}\left(X_{i} \sqcup Y_{i}\right) .
$$


Proof. It is sufficient to define a composition for elements $f \in \mathbb{P}(\mathcal{M})(X, Y)$ of the special type $f=\alpha \sqcup I$, where $X=X^{\prime} \sqcup X^{\prime \prime}, \quad Y=Y^{\prime} \sqcup Y^{\prime \prime}$, $I=\sqcup_{x \in X^{\prime \prime}} I_{x, \phi(x)}, \quad \phi: X^{\prime \prime} \rightarrow Y^{\prime \prime}$ is a bijection, and $\alpha \in \mathcal{M}\left(X^{\prime} \sqcup Y^{\prime}\right)$.

Composition of elements of this type is defined using the composition in the modular operad $\mathcal{M}$.

3.4.2. From modular to cyclic operads. If $\mathcal{M}$ is a modular operad, its zeroth component $\mathcal{M}^{0}$ is a cyclic operad. This gives a functor

$$
\mathrm{Gr}^{0}: \text { ModOp } \rightarrow \text { CycOp. }
$$

For non-graded modular operads there is a natural forgetful functor

$$
\#_{n g r}: \mathrm{ModOp}^{n g r} \rightarrow \mathrm{CycOp}: \quad A \mapsto A^{\#} .
$$

3.4.3. From non-graded modular operads to graded and back. There exists a pair of adjoint functors

$$
\text { Tot }: \text { ModOp } \rightarrow \text { ModOp }{ }^{n g r}
$$

and

$$
\text { Const }: \text { ModOp }{ }^{n g r} \rightarrow \text { ModOp }
$$

defined by the following formulas

$$
\operatorname{Tot}\left(\left\{\mathcal{M}^{n}\right\}\right)=\bigoplus_{n} \mathcal{M}^{n} \text { and } \operatorname{Const}(\mathcal{M})^{n}=\mathcal{M}
$$

The composition $\mathrm{Gr}^{0} \circ$ Const is isomorphic to the forgetful functor $\#_{n g r}$ (20).

\subsection{Augmented cyclic and modular operads.}

We will need the following variation of the notions of cyclic and modular operads.

3.5.1. Definition. An augmented cyclic operad is a functor on the groupoid of finite sets

$$
\mathcal{O}: \mathbb{S} \rightarrow \text { Vect, }
$$

endowed with a collection of compositions

$$
\circ_{x y}: \mathcal{O}(X) \otimes \mathcal{O}(Y) \rightarrow \mathcal{O}(X \sqcup Y \backslash\{x, y\})
$$

defined for each pair $x \in X, y \in Y$ satisfying the commutativity, associativity, and unity conditions (i), (ii), and (iii) of 3.2.3. 
3.5.2. Remark. To define a structure of augmented cyclic operad on a cyclic operad $\mathcal{O}$, one has to choose a graded vector space $\mathcal{O}(\emptyset)$ and to define an operation

$$
\circ_{x y}: \mathcal{O}(\{x\}) \otimes \mathcal{O}(\{y\}) \longrightarrow \mathcal{O}(\emptyset) .
$$

In particular, every cyclic operad can be considered as an augmented cyclic operad with $\mathcal{O}(\emptyset)=0$.

3.5.3. Definition. An augmented modular operad is a collection of functors $\mathcal{M}^{n}: \mathbb{S} \rightarrow$ Vect, $n=0,1, \ldots$, with a collection of compositions

$$
\circ_{f}: \mathcal{M}^{m}(X) \otimes \mathcal{M}^{n}(Y) \rightarrow \mathcal{M}^{m+n+d-1}(X \sqcup Y \backslash(U \sqcup V))
$$

defined for each bijection $U \stackrel{f}{\longrightarrow} V$ between non-empty $d$-element subsets $U \subset X$ and $V \subset Y$ satisfying the conditions (i)-(iii) of 3.3.1.

The category of augmented operads will be denoted ModOp . $^{\text {. }}$

The following is a version of Proposition 3.3.2 for augmented modular operads.

3.5.4. Proposition. An augmented modular operad can be defined as an augmented graded cyclic operad

$$
\mathcal{M}^{n}: \mathbb{S} \rightarrow \text { Vect }, \quad n=0,1, \ldots,
$$

endowed with contraction operations

$$
c_{x y}: \mathcal{M}^{n}(X) \rightarrow \mathcal{M}^{n+1}(X \backslash\{x, y\}), \quad x, y \in X, x \neq y,
$$

satisfying properties (i)-(iii) of Proposition 3.3.2, where in property (iii) we do not require that $X \sqcup Y \neq\left\{x_{1}, x_{2}, y_{1}, y_{2}\right\}$.

3.5.5. PROP from an augmented modular operad. Similarly to 3.4.1, we consider a functor $\mathbb{P}_{+}$that associates a PROP to an augmented modular operad.

Let $\mathcal{M}$ be an augmented modular operad. Define a collection of vector spaces $\mathbb{P}_{+}(\mathcal{M})(X, Y)$ for $X, Y \in \mathbb{S}$ by the formula

$$
\mathbb{P}_{+}(\mathcal{M})(X, Y)=S(\mathcal{M}(\emptyset)) \otimes\left(\bigoplus_{\substack{X \\ Y=\coprod_{i \in I} X_{i} \\ X_{i} \sqcup \coprod_{i} \in Y_{i}}} \bigotimes_{i \in I} \mathcal{M}\left(X_{i} \sqcup Y_{i}\right)\right),
$$

where $S(V)$ is the symmetric algebra of the vector space $V$. 
An argument similar to the proof of Proposition 3.4.1 shows that the assignment $\mathcal{M} \mapsto \mathbb{P}_{+}(\mathcal{M})$ gives a functor

$$
\mathbb{P}_{+}: \text {ModOp }+\rightarrow \text { Props. }
$$

We will also need the following forgetful functor

$$
\#: \operatorname{ModOp}_{+} \rightarrow \operatorname{ModOp}: \quad \mathcal{M} \mapsto \mathcal{M}^{\#}
$$

given by the formula

$$
\mathcal{M}^{\#}(X)=\mathcal{M}(X), \text { for } X \neq \emptyset
$$

3.5.6. Comparison with the definition of GKM]. The modular operads of Getzler-Kapranov [GKM] are in our terminology augmented modular operads $\mathcal{M}$ satisfying the following additional stability requirements:

- $\mathcal{M}^{0}(\emptyset)=\mathcal{M}^{1}(\emptyset)=0$;

- $\mathcal{M}^{0}(\{x\})=0$

- $\mathcal{M}^{0}(\{x, y\})=k \cdot I_{x y}$.

\subsection{Results.}

In this section we formulate the main results of the first part of the paper. They claim the existence of some adjoint functors to the standard functors defined in 3.4 and 3.5 together with various relations between these functors. Proofs of these results will be given in the next section.

The following diagram shows relevant categories and functors. The triangles formed by solid arrows will be commutative.

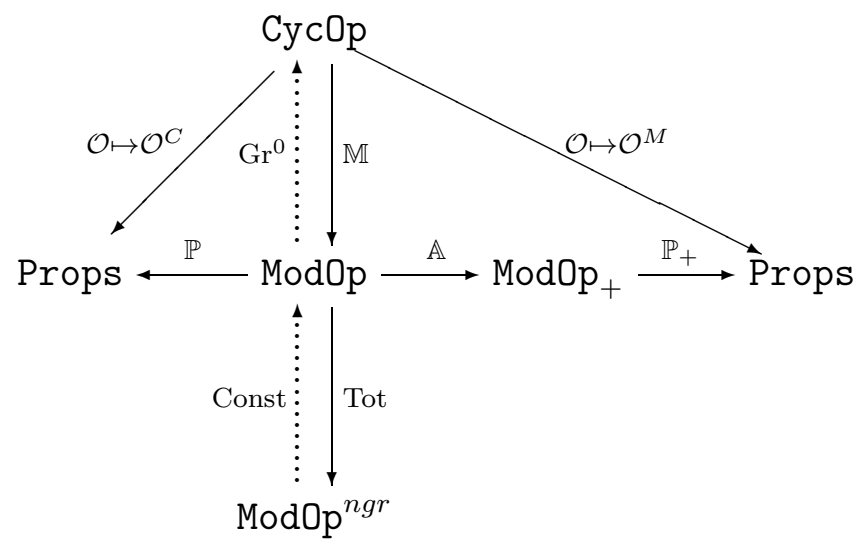


3.6.1. Theorem. The zero-component functor (19)

$$
\mathrm{Gr}^{0}: \mathrm{ModO} \rightarrow \mathrm{CycOp}
$$

admits a left adjoint functor

$$
\mathbb{M}: \text { CycOp } \rightarrow \text { ModOp. }
$$

\subsubsection{Corollary. The functor}

$$
\text { Tot } \circ \mathbb{M}: \text { CycOp } \rightarrow \text { ModOp }^{n g r}
$$

is a left adjoint to the forgetful functor (20)

$$
\#_{n g r}: \text { ModOp }^{n g r} \rightarrow \text { CycOp. }
$$

3.6.3. Remark. Let $V: \mathbb{S}^{*} \rightarrow$ Vect be a functor and $F(V)$ be the free cyclic operad generated by $V$ (see GKQ). Since by Theorem 3.6.1 the functor $\mathbb{M}$ is left adjoint to the zero-component functor $\mathrm{Gr}_{0}$ and the functor $F: V \mapsto F(V)$ is left adjoint to the forgetful functor $\#_{n g r}$, the functor $\mathbb{M} \circ F$ is left adjoint to the composition of the functor $\#_{n g r}$ with $\mathrm{Gr}^{0}$. This shows that $\mathbb{M}(F(V))$ can be considered as the free modular operad generated by $V$ (see GKM).

3.6.4. Theorem. The functors $\mathbb{P} \circ \mathbb{M}$ and $\mathcal{O} \mapsto \mathcal{O}^{C}$ from Cyc0p to Props are isomorphic, i.e. the left solid triangle of the diagram (25) is commutative.

3.6.5. Theorem. The forgetful functor

$$
\#: \text { ModOp }+ \text { ModOp }
$$

admits a left adjoint

$$
\mathbb{A}: \text { ModOp } \rightarrow \text { ModOp }+
$$

3.6.6. Theorem. Let $\mathbb{M}_{+}$denote the composition of functors $\mathbb{A} \circ \mathbb{M}$.

The functors $\mathbb{P}_{+} \circ \mathbb{M}_{+}$and $\mathcal{O} \mapsto \mathcal{O}^{M}$ from Cyc0p to Props are isomorphic. In other words, the right solid triangle of the diagram 25 is commutative.

3.6.7. Corollary. Let $\mathcal{O}$ be a cyclic operad. For each $X \in \mathbb{S}^{*}$ there exists a natural isomorphism of vector spaces

$$
\mathcal{O}^{M}(\emptyset, \emptyset) \otimes \mathcal{O}^{C}(\emptyset, X) \stackrel{\sim}{\longrightarrow} \mathcal{O}^{M}(\emptyset, X) .
$$


CYCLIC OPERADS AND CHORD DIAGRAMS

3.6.8. Corollary. Let $\mathcal{O}$ be a cyclic operad. The natural map

$$
\mathcal{O}^{C}(0, n) \rightarrow \mathcal{O}^{M}(0, n)
$$

is injective for all $n \geq 0$.

\section{Proofs}

In this section we prove the results formulated in Sections 3.6.1 3.6.8. The technical heart of the proof is the fact that the operadic part of the PROP $\mathcal{O}^{C}$ admits a natural structure of a modular operad. This is established in 4.1 .

Then in 4.2 we show how to deduce the statements of 3.6.1 - 3.6.8 from this fact.

\subsection{A modular operad from a cyclic operad.}

Let $\mathcal{O}$ be a cyclic operad. Consider a family of vector spaces labelled by pairs $(X, x), \quad X \in \mathbb{S}^{*}, \quad x \in X$ given by

$$
\mathcal{M}_{x}(X)=\mathcal{O}^{C}(X \backslash\{x\},\{x\}),
$$

where the right-hand side is understood in the PROP sense.

This is just the operadic part of the $\operatorname{PROP} \mathcal{O}^{C}$.

Our goal is to introduce a structure of a modular operad on this collection of spaces $\mathcal{M}$. In particular we will canonically identify $\mathcal{M}_{x}(X)$ for different $x \in X$. The resulting modular operad will be denoted $\mathbb{M}(\mathcal{O})$.

4.1.1. Grading. Recall that $\mathcal{O}^{C}$ is defined in 3.1.6 as the PROP generated over $\mathrm{P}(\mathcal{O})$ by the casimir $c \in \mathcal{O}^{C}$, subject to relations $(10)$. These relations are homogeneous with respect to the number of casimirs, therefore, the space $\mathcal{M}_{x}(X)$ obtains a natural grading

$$
\mathcal{M}_{x}(X)=\bigoplus_{n \geq 0} \mathcal{M}_{x}^{n}(X)
$$

where $\mathcal{M}_{x}^{n}(X)$ is the space generated by the compositions

$$
f \circ c^{\otimes n}
$$

where $c^{\otimes n}: \emptyset \rightarrow Y \sqcup Y^{\prime}$, with $|Y|=\left|Y^{\prime}\right|=n$ and $f \in \mathcal{O}\left(X \sqcup Y \sqcup Y^{\prime}\right)$.

Note that since the presentation (30) is not unique, we cannot use it to identify $\mathcal{M}_{x}(X)$ for different $x$. The degree zero part of $\mathcal{M}_{x}(X)$ coincides with $\mathcal{O}(X)$.

We will introduce the structure of a modular operad on the collection of spaces $\mathcal{M}_{x}^{n}(X)$ by induction on degree. 
For a pointed set $(X, x)$ denote by $\widehat{X}$ the set $X \sqcup\left\{y, y^{\prime}\right\}$ and define a morphism

$$
c=c_{y y^{\prime}}: \mathcal{M}_{x}^{n}(\widehat{X}) \rightarrow \mathcal{M}_{x}^{n+1}(X)
$$

as the composition with the casimir $c_{y y^{\prime}} \in \mathcal{O}^{C}\left(\emptyset,\left\{y, y^{\prime}\right\}\right)$ in the PROP $\mathcal{O}^{C}$.

\subsubsection{Lemma.}

1. The map $c_{y y^{\prime}}$ is a surjection for $n \geq 0$.

2. Suppose that $n \geq 1$ and put $\widehat{\widehat{X}}=X \sqcup\left\{y, y^{\prime}, z, z^{\prime}\right\}$. In the sequence

$$
\mathcal{M}_{x}^{n-1}(\widehat{\widehat{X}}) \underset{c_{2}}{\stackrel{c_{1}}{\longrightarrow}} \mathcal{M}_{x}^{n}(\widehat{X}) \stackrel{c}{\longrightarrow} \mathcal{M}_{x}^{n+1}(X)
$$

the compositions $c c_{1}$ and $c c_{2}$ coincide.

Here

$$
c_{1}=c_{z z^{\prime}} \text { and } c_{2}=\theta c_{y y^{\prime}}
$$

where $\theta$ is induced by the involution of $\widehat{\widehat{X}}$ identical on $X$ and sending $y$ to $z$ and $y^{\prime}$ to $z^{\prime}$.

Proof. Straightforward from the definition of the spaces $\mathcal{M}_{x}^{n}(X)$.

The following is the key technical result of this section.

4.1.3. Proposition. The collection of vector spaces $\mathcal{M}_{x}(X)$ admits a natural structure of a modular operad.

We will construct all necessary structures by induction on degree. Simultaneously with checking the necessary properties we will establish the following characterization of the kernel of the morphism (31).

4.1.4. Lemma. Let $k \geq 0$ and $x \in X$. The kernel of the map

$$
c: \mathcal{M}_{x}^{k}(\widehat{X}) \rightarrow \mathcal{M}_{x}^{k+1}(X)
$$

is generated by the following three types of elements:

(i) $\quad \alpha+\sigma_{y y^{\prime}}(\alpha)$,

where $\alpha \in \mathcal{M}^{k}(\widehat{X})$ and $\sigma_{y y^{\prime}}$ is the automorphism of $\widehat{X}$ interchanging $y$ and $y^{\prime}$;

(ii) $\quad \alpha \circ_{z z^{\prime}} \beta-f_{*}\left(\alpha \circ_{y y^{\prime}} \beta\right)$, where $X=A^{\circ} \sqcup B^{\circ} ; A=A^{\circ} \sqcup\{y, z\} ; B=B^{\circ} \sqcup\left\{y^{\prime}, z^{\prime}\right\} ; \alpha \in \mathcal{M}^{k_{1}}(A)$; $\beta \in \mathcal{M}^{k_{2}}(B) ; k=k_{1}+k_{2}$; and $f: X \sqcup\left\{z, z^{\prime}\right\} \rightarrow X \sqcup\left\{y, y^{\prime}\right\}$ is the bijection identical on $X$ and sending $z$ to $y$ and $z^{\prime}$ to $y^{\prime}$; 
(iii) $\quad c_{1}(\alpha)-c_{2}(\alpha)$,

where $\alpha \in \mathcal{M}_{x}^{k}\left(X \sqcup\left\{y, y^{\prime}, z, z^{\prime}\right\}\right), \quad k \geq 1$, and the maps $c_{1}$ and $c_{2}$ are given in (33).

It is easy to see that any element of one of the types (i)-(iii) belongs to $\operatorname{Ker}(c)$. For type (i) this is so because $c$ is symmetric, for type (ii) it follows from the invariance property (10) of $c$, and for (iii) this is the statement 2 of Lemma 4.1.2.

Therefore, to prove Lemma 4.1.4 it remains to show that any element of $\operatorname{Ker}(c)$ is a combination of elements of these three types. We will prove this by induction on $k$. Before making the $k$-th step we will identify all the spaces $\mathcal{M}_{x}^{k}(X)$ for different $x \in X$.

4.1.5. Induction hypothesis. Let us assume that we have the necessary structure on $\mathcal{M}^{k}$ for all $k \leq n$. This includes the following components.

1. A canonical identification of $\mathcal{M}_{x}^{k}(X)$ for different $x \in X$ (which makes $\mathcal{M}^{k}(X)$ well-defined) for every $k \leq n$. This means that a compatible collection of isomorphisms

$$
\phi_{*}: \mathcal{M}_{x}^{k}(X) \rightarrow \mathcal{M}_{\phi(x)}^{k}(X)
$$

is given for each automorphism $\phi: X \rightarrow X$.

We assume that the maps (31) $c: \mathcal{M}^{k}(\widehat{X}) \rightarrow \mathcal{M}^{k+1}(X)$ are equivariant with respect to automorphisms of $X$ for $k+1 \leq n$.

2. A collection of functorial operations

$$
\circ_{f}: \mathcal{M}^{p}(X) \otimes \mathcal{M}^{q}(Y) \rightarrow \mathcal{M}^{p+q+d-1}(X \sqcup Y \backslash(U \sqcup V)),
$$

defined for bijections $f: U \stackrel{\sim}{\longrightarrow} V$, where $d=|U|=|V| \geq 1$ and $p+q+d-1 \leq n$. These operations satisfy conditions (i)-(iii) of definition 3.3.1 when all the compositions make sense.

3. The statement of Lemma 4.1.4 is valid for all $0 \leq k<n$.

After completing the induction step we will have constructed this data for $k=n+1$.

Let us verify the base of induction.

4.1.6. Tree level case: $n=0$. We have $\mathcal{M}_{x}^{0}(X)=\mathcal{O}(X)$, therefore there is a well-defined cyclic structure on $\mathcal{M}^{0}$. In particular, it gives a canonical identification of spaces $\mathcal{M}_{x}^{0}(X)$ for different $x \in X$. The structure of a cyclic operad on $\mathcal{M}^{0}$ also gives all composition operations with values in $\mathcal{M}^{0}$. The statement of Lemma 4.1.4 is empty for $k<0$. 
4.1.7. Induction step. Suppose that we have the structure elements (1)-(3) of 4.1.5 on $\mathcal{M}^{k}$ for $k \leq n$. Now we are going to extend this data to $\mathcal{M}^{n+1}$.

First in 4.1.8 4.1.10 we will prove the key Lemma 4.1.4 for $k=n$. After that we will finish the induction step in 4.1.11.

4.1.8. Beginning of the induction step for Lemma 4.1.4. In order to prove Lemma 4.1.4 for $k=n$ we first define a collection of functors

$$
\mathcal{T}^{k}: \mathbb{S}^{*} \rightarrow \text { Vect }
$$

by the formula

$$
\mathcal{T}^{k}(X)= \begin{cases}0 & \text { if } k>n+1 \\ \mathcal{M}^{k}(X) & \text { if } k \leq n \\ \mathcal{M}^{n}(\widehat{X}) / R & \text { if } k=n+1\end{cases}
$$

where $R$ is the subspace of $\mathcal{M}(\widehat{X})$ generated by the elements (i) - (iii) of 4.1.4.

4.1.9. Lemma. The set of functors $\mathcal{T}=\left\{\mathcal{T}^{k}\right\}$ admits a natural structure of a modular operad.

Proof. We will use here the definition of modular operad given by Proposition 3.3.2. Functoriality of $\mathcal{T}(X)$ with respect to $X$ follows from its definition. It only remains to construct the composition and contraction operations.

By the induction hypothesis, it only remains to define operation and contractions with values in $\mathcal{T}^{n+1}$. Therefore, we need to define operations of the following three kinds.

(A) $\alpha \circ \beta$ for $\alpha \in \mathcal{T}^{n+1}, \quad \beta \in \mathcal{T}^{0}$;

(B) $\quad \alpha \circ \beta$ for $\alpha \in \mathcal{T}^{k}, \quad \beta \in \mathcal{T}^{n+1-k}, \quad 1 \leq k \leq n$;

(C) $\quad c_{x y}(\alpha)$ for $\alpha \in \mathcal{T}^{n}(X), \quad x, y \in X, \quad X \neq\{x, y\}$.

Definition of operations of type $(A)$.

Take any $\tilde{\alpha} \in \mathcal{M}^{n}(\widehat{X})$ representing $\alpha$ and define $\alpha \circ \beta$ as the image of $\tilde{\alpha} \circ \beta$ in $\mathcal{T}^{n+1}$.

This is well-defined since the subspace $R$ generated by the relations (i) - (iii) is stable under multiplication by $\beta$.

Definition of operations of type $(B)$.

Take $\tilde{\alpha} \in c^{-1}(\alpha) \in \mathcal{M}^{k-1}(\widehat{X})$ and define $\alpha \circ \beta$ to be the image of the composition $\tilde{\alpha} \circ \beta$ which is defined by the induction hypothesis.

Definition of operations of type $(C)$.

The map $c_{x y}: \mathcal{T}^{n}(X) \rightarrow \mathcal{T}^{n+1}(X \backslash\{x, y\})$ is obtained from the definition of $\mathcal{T}^{n+1}(X \backslash\{x, y\})$ by identification (any one) of $X$ with 
$X \widehat{\backslash\{x, y\}}$. The result does not depend on the identification because the difference is an element of type (i) in the subspace $R$.

Let us verify that these operations define a structure of a modular operad on $\mathcal{T}$. This amounts to checking the following properties.

0. $c_{x y}=c_{y x}$.

This is one of the defining properties of $c$.

1. Independent contractions commute.

This is so because their difference in $\mathcal{M}^{n}(\widehat{X})$ is an element of type (iii) in the subspace $R$ and therefore vanishes in $\mathcal{T}^{n+1}$.

2. Contractions commute with compositions - property (3) of 3.3.2.

This is true because in $\mathcal{M}^{n}(\widehat{X})$ the difference of the corresponding elements is an element of $R$ of type (ii).

3. Commutativity of the composition.

Here we need to check that for $\alpha \in \mathcal{T}^{k}, \beta \in \mathcal{T}^{n+1-k}, 1 \leq k \leq n$, the difference $\tilde{\alpha} \circ \beta-\alpha \circ \tilde{\beta}$ belongs to $R$. Indeed, this difference can be written as $\left(c_{1}-c_{2}\right)(\tilde{\alpha} \circ \tilde{\beta})$ in the notation of $(33)$ and therefore is an element of $R$ of type (iii).

Note that for $\alpha \in \mathcal{T}^{k}, \beta \in \mathcal{T}^{l}$, with $1 \leq k \leq n-l$, one also has

$$
\alpha \circ \beta=c(\tilde{\alpha} \circ \beta) \text {. }
$$

4. Associativity of the composition.

We need to show that when $\operatorname{deg} \alpha+\operatorname{deg} \beta+\operatorname{deg} \gamma=n+1$ the composition $\alpha \circ \beta \circ \gamma$ is well-defined.

Since the sum of the degrees of $\alpha, \beta$, and $\gamma$ is positive, at least one of these elements has a positive degree. In the case when $\operatorname{deg} \alpha>0$, the element $\alpha \circ(\beta \circ \gamma) \in \mathcal{T}^{n+1}$ is the image of

$$
\tilde{\alpha} \circ(\beta \circ \gamma)=(\tilde{\alpha} \circ \beta) \circ \gamma \in \mathcal{M}^{n} .
$$

There may be two possibilities: $\operatorname{deg} \gamma=0$ and $\operatorname{deg} \gamma>0$.

In the case when $\operatorname{deg} \gamma=0$, the composition $\alpha \circ \beta$ belongs to $\mathcal{T}^{n+1}$ and is represented in $\mathcal{M}^{n}$ by $\tilde{\alpha} \circ \beta$. Therefore by definition of the operation of type $(\mathrm{A})$ the composition $(\alpha \circ \beta) \circ \gamma$ is represented by $(\tilde{\alpha} \circ \beta) \circ \gamma \in \mathcal{M}^{n}$.

If $\operatorname{deg} \gamma>0$, one still has

$$
\alpha \circ \beta=c(\tilde{\alpha} \circ \beta)
$$

and by definition of the operation of type (B) we get the same result.

The cases when $\operatorname{deg} \beta>0$ or $\operatorname{deg} \gamma>0$ are considered similarly.

This concludes the construction of the modular operad structure on $\mathcal{T}$. 
4.1.10. End of induction step for Lemma 4.1.4. Consider PROP $\mathbb{P}(\mathcal{T})$ generated by the modular operad $\mathcal{T}$ (see 3.4.1).

By definition of $\mathcal{T}$ there is a natural morphism

$$
\mathcal{O} \rightarrow \mathcal{T}
$$

and therefore, by the universal property of $\mathcal{O}^{C}$, we have a morphism of PROPs

$$
f: \mathcal{O}^{C} \rightarrow \mathbb{P}(\mathcal{T})
$$

Morphism $f$ is a surjection - it is an isomorphism in degree zero and the maps $\mathcal{T}^{k}(\widehat{X}) \rightarrow \mathcal{T}^{k+1}(X)$ are surjective for $k \leq n-1$ by Lemma 4.1.2, and for $k=n$ by definition of $\mathcal{T}^{n+1}$.

On the other hand, we have a natural morphism of vector spaces $\mathcal{T}^{n+1}(X) \rightarrow \mathcal{M}_{x}^{n+1}(X)$ induced by the map $c$ (31). This morphism splits the morphism $\mathcal{O}^{C}(X \backslash\{x\}, x) \rightarrow \mathcal{P}$ which proves Lemma 4.1.4 for $k=n$.

4.1.11. End of the proof of Proposition 4.1.3. Using Lemma 4.1.4 for $k=n$, we can easily complete the induction step.

First, since the kernel of $c: \mathcal{M}_{x}^{n}(\widehat{X}) \rightarrow \mathcal{M}_{x}^{n+1}(X)$ is invariant under the action of the group of automorphisms of $X$, it induces a natural isomorphism between $\mathcal{M}_{x}^{n+1}(X)$ for different $x \in X$. We also have to define new operations in $\mathcal{M}$ with values in $\mathcal{M}^{n+1}$, but this has already been done - we defined them for the modular operad $\mathcal{T}$ which coincides with $\mathcal{M}$ in degrees $\leq n+1$.

Proposition 4.1 .3 is proved.

\subsection{Proof of $3.6 .1-3.6 .8$.}

4.2.1. Proof of Theorem 3.6.4. By construction of $\mathbb{P}(\mathbb{M}(\mathcal{O}))$ we have a natural morphism $\mathcal{O} \rightarrow \mathbb{P}(\mathbb{M}(\mathcal{O}))$. The universal property of $\mathcal{O}^{C}$ yields a map of PROPs

$$
F: \mathcal{O}^{C} \rightarrow \mathbb{P}(\mathbb{M}(\mathcal{O}))
$$

that sends the casimir of $\mathcal{O}^{C}$ to the element $c \in \mathbb{P}(\mathbb{M}(\mathcal{O}))(\emptyset,\{x, y\})$ corresponding to the identity element $I_{x y}$.

The inverse map $G: \mathbb{P}(\mathbb{M}(\mathcal{O})) \rightarrow \mathcal{O}^{C}$ is constructed as follows. For a pair of sets $X$ and $Y \neq \emptyset$ and an element $f \in \mathcal{O}^{C}(X \sqcup Y \backslash\{y\}, y)$ let $Y^{\prime}$ be a disjoint from $Y$ copy of $Y \backslash\{y\}$ with a fixed bijection $j: Y \backslash\{y\} \stackrel{\sim}{\longrightarrow} Y^{\prime}$ and define $G(f)$ as the composition

$$
X \stackrel{\operatorname{id}_{X} \sqcup c_{Y}}{\longrightarrow} X \sqcup(Y \backslash\{y\}) \sqcup Y^{\prime} \stackrel{f \sqcup \operatorname{id}_{Y^{\prime}}}{\longrightarrow}\{y\} \sqcup Y^{\prime} \stackrel{\sim}{\longrightarrow} Y,
$$


This defines a collection of maps $\mathbb{M}(\mathcal{O})(X \sqcup Y) \rightarrow \mathcal{O}^{C}(X, Y)$ that automatically extends to a map of PROPs

$$
G: \mathbb{P}(\mathbb{M}(\mathcal{O})) \rightarrow \mathcal{O}^{C}
$$

inverting the map $F: \mathcal{O} \rightarrow \mathbb{P}(\mathbb{M}(\mathcal{O}))$.

4.2.2. Proof of Theorem 3.6.1. The composition $\mathcal{O} \mapsto \mathbb{M}(\mathcal{O}) \mapsto \mathbb{M}(\mathcal{O})^{0}$ is the identity.

Let $\mathcal{M}$ be a modular operad. We need to construct a map in the opposite direction $\eta: \mathbb{M}\left(\mathcal{M}^{0}\right) \rightarrow \mathcal{M}$ functorial in $\mathcal{M}$.

We construct $\eta^{k}: \mathbb{M}\left(\mathcal{M}^{0}\right)^{k} \rightarrow \mathcal{M}^{k}$ by induction on $k$. In the case $k=0$ these spaces are the same and the map $\eta^{0}$ is the identity. For $k \geq 0$ consider a diagram

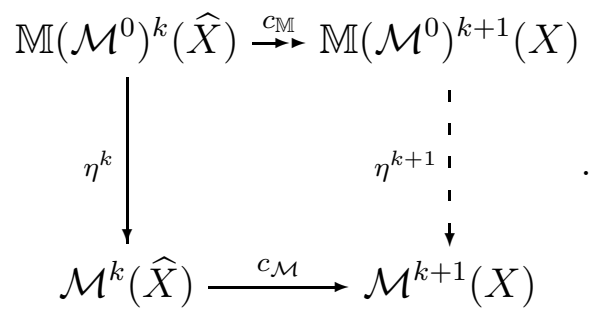

The map $c_{\mathbb{M}}$ is a surjection and Lemma 4.1.4 implies that $\eta^{k}\left(\operatorname{Ker}\left(c_{\mathbb{M}}\right)\right)$ lies in $\operatorname{Ker}\left(c_{\mathcal{M}}\right)$. This allows us to extend uniquely $\eta^{k}$ to $\eta^{k+1}$.

4.2.3. Proof of Theorem 3.6.5. Let $\mathcal{M}$ be a modular operad. Define $\mathcal{A}=\mathbb{A}(\mathcal{M}) \in$ ModOp $_{+}$as follows.

Put $\mathcal{A}(X)=\mathcal{M}(X)$ if $X \neq \emptyset$. Furthermore, put

$$
\mathcal{A}^{0}(\emptyset)=S^{2}\left(\mathcal{M}^{0}(\{x\})\right)
$$

so that the only composition with values in $\mathcal{A}^{0}(\emptyset)$,

$$
\circ_{x y}: \mathcal{A}^{0}(\{x\}) \otimes \mathcal{A}^{0}(\{y\}) \rightarrow \mathcal{A}^{0}(\emptyset),
$$

is defined.

Finally, define $\mathcal{A}^{n+1}(\emptyset)$ as the quotient $\mathcal{M}^{n}(\{x, y\}) / R$, where, as in 4.1.8, the subspace $R$ is generated by the elements (i) - (iii) of 4.1.4.

To define a structure of an augmented modular operad on $\mathcal{A}$ we will use the second definition 3.5.4. The only missing operation is the contraction

$$
c_{x, y}: \mathcal{A}^{n}(\{x, y\}) \rightarrow \mathcal{A}^{n+1}(\emptyset)
$$

and we define it as the natural projection of $\mathcal{A}^{n}(\{x, y\})$ to its quotient. The properties (i) - (iii) of Definition 3.3 .2 can be easily verified. 
4.2.4. Proof of Theorem 3.6.0. By definition of $\mathbb{P}_{+}\left(\mathbb{M}_{+}(\mathcal{O})\right)$ we have a natural morphism $\mathcal{O} \rightarrow \mathbb{P}_{+}\left(\mathbb{M}_{+}(\mathcal{O})\right)$. The universal property of $\mathcal{O}^{M}$ gives a map of PROPs

$$
F: \mathcal{O}^{M} \rightarrow \mathbb{P}_{+}\left(\mathbb{M}_{+}(\mathcal{O})\right)
$$

as follows. The map $F$ sends the casimir of $\mathcal{O}^{M}$ to the element $c \in$ $\mathbb{P}_{+}\left(\mathbb{M}_{+}(\mathcal{O})\right)(\emptyset,\{x, y\})$ corresponding to the identity element $I_{x y}$.

Similarly, $F$ sends the metric $b \in \mathcal{O}^{M}(2,0)$ to the element

$$
b \in \mathbb{P}_{+}\left(\mathbb{M}_{+}(\mathcal{O})\right)(\{x, y\}, \emptyset)
$$

corresponding to the identity element $I_{x y}$.

The inverse map is constructed as follows. We start with the collection of maps

$$
\mathbb{M}(\mathcal{O})(X \sqcup Y) \rightarrow \mathcal{O}^{C}(X, Y) \rightarrow \mathcal{O}^{M}(X, Y), \quad Y \neq \emptyset,
$$

defined in 4.2.1, and extend it to a larger collection

$$
G(X, Y): \mathbb{M}_{+}(\mathcal{O})(X \sqcup Y) \rightarrow \mathcal{O}^{M}(X, Y),
$$

where $X$ and $Y$ may be empty. Namely, if $Y=\emptyset, X \neq \emptyset$, we define the map $G(X, \emptyset)$ as the composition

$$
\mathbb{M}_{+}(\mathcal{O})(X)=\mathbb{M}(\mathcal{O})(X) \rightarrow \mathcal{O}^{M}(\emptyset, X) \stackrel{s}{\rightarrow} \mathcal{O}^{M}(X, \emptyset),
$$

where the map $s$ is the composition with the element

$$
b^{\otimes n} \in \mathcal{O}^{M}\left(X \sqcup X^{\prime}, \emptyset\right), n=|X|=\left|X^{\prime}\right| .
$$

Finally, the map

$$
G(\emptyset, \emptyset): \mathbb{M}_{+}(\mathcal{O})(\emptyset) \rightarrow \mathcal{O}^{M}(\emptyset, \emptyset)
$$

in positive degrees is defined as the composition of $G(\{x, y\}, \emptyset)$ with the contraction operation in $\mathbb{M}_{+}(\mathcal{O})$ and $c \in \mathcal{O}^{M}(\emptyset,\{x, y\})$.

4.2.5. Proof of 3.6. and 3.6.8. Using the explicit definition 3.5.5 of the PROP generated by an augmented modular operad, we obtain Corollary 3.6.7.

Corollary 3.6 .8 is its immediate consequence. 


\section{ENVELOPING ALGEBRAS}

In this section we discuss different approaches to defining a universal enveloping algebra of an algebra over an operad in a tensor category. We show that in the case of Lie algebras both definitions of an external universal algebra are equivalent under mild assumptions on the tensor category. In this case we also study the Hopf algebra structure and the notion of a center of an enveloping algebra.

In Section 7 we will show that the algebra $\mathcal{A}$ of chord diagrams arising in the theory of Vassiliev knot invariants is isomorphic to the enveloping algebra of the universal Casimir Lie algebra $\mathbb{L}^{C} \in \operatorname{LIE}^{C}$.

5.1. Definitions of enveloping algebras. The following is a standard definition of the enveloping algebra of an algebra over an operad in a tensor category (see, e.g., [HS], Sect. 3). Note that it defines an internal enveloping algebra, i.e. an associative algebra in the tensor category.

5.1.1. Definition. Let $\mathcal{O}$ be an operad and $A$ be an $\mathcal{O}$-algebra in a tensor category $\mathcal{C}$. An associative algebra $\mathcal{U}$ in $\mathcal{C}$ endowed with a collection of maps

$$
u_{n}: \mathcal{O}(n+1) \otimes A^{\otimes n} \rightarrow \mathcal{U},
$$

$n=1,2, \ldots$, is called an internal enveloping algebra of $A$ if $u_{n}$ is $\Sigma_{n^{-}}$ equivariant, the following diagram

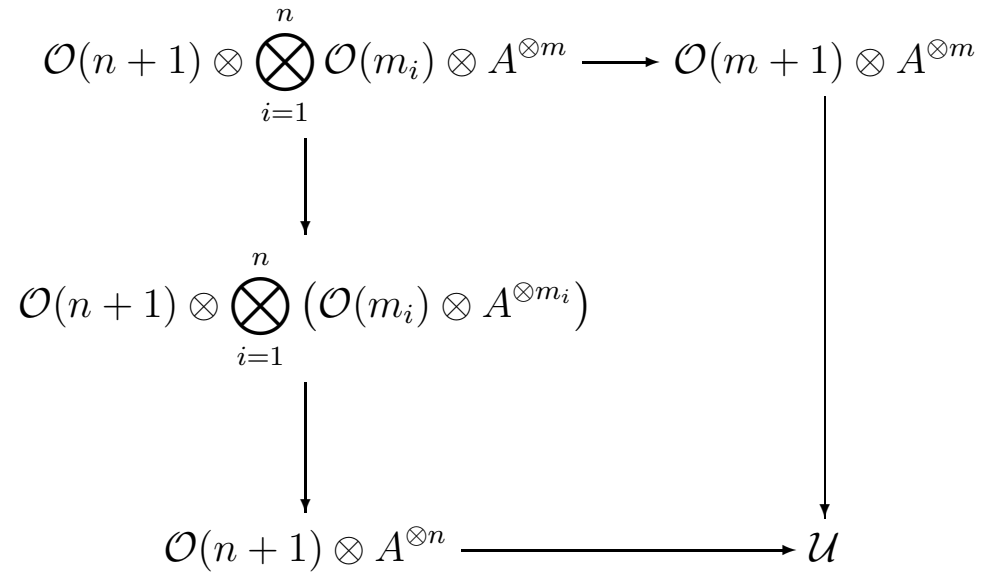

is commutative for all $n, m_{1}, \ldots, m_{n}$ and $m=m_{1}+\ldots+m_{n}$, and $\mathcal{U}$ is universal with respect to these properties.

An internal universal algebra of an $\mathcal{O}$-algebra $A$, if it exists, is unique up to an isomorphism and will be denoted by $\mathcal{U}(\mathcal{O}, A)$ or sometimes simply by $\mathcal{U}(A)$. 
Internal enveloping algebras exist, for example, when the tensor category $\mathcal{C}$ admits colimits. In this case the enveloping algebra $\mathcal{U}(\mathcal{O}, A)$ can be described as the quotient of the internal tensor algebra

$$
\mathcal{T}(\mathcal{O}, A)=\bigoplus_{n \in \mathbb{N}} \mathcal{O}(n+1) \otimes_{\Sigma_{n}} A^{\otimes n}
$$

by an ideal defined in a usual way.

Let $\mathcal{O}$ be an operad and $\mathcal{C}$ be a tensor category. We give two different definitions of an external enveloping algebra of an $\mathcal{O}$-algebra $A$ in $\mathcal{C}$. These algebras are ordinary associative algebras in the category Vect. In general they may not be isomorphic, but in the important for us case of the operad $\mathcal{O}=$ LIE these definitions are equivalent under some mild conditions.

Denote by $\Gamma$ the global section functor

$$
\Gamma: \mathcal{C} \rightarrow \operatorname{Vect}, \quad \Gamma(X)=\operatorname{Hom}(\mathbb{I}, X) .
$$

5.1.2. Definition. Suppose that the internal enveloping algebra $\mathcal{U}(\mathcal{O}, A)$ exists. Then the global enveloping algebra $\mathfrak{U}(\mathcal{O}, A)$ of $A$ is defined as

$$
\mathfrak{U}(\mathcal{O}, A)=\Gamma(\mathcal{U}(\mathcal{O}, A))
$$

with operations induced by the structure of an associative algebra in $\mathcal{C}$ on $\mathcal{U}(\mathcal{O}, A)$.

Another way to construct an external enveloping algebra of $A$ is to start with the external tensor algebra of $A$ and then consider an appropriate quotient.

The external tensor algebra of an $\mathcal{O}$-algebra $A$ is defined by the following formula

$$
T(\mathcal{O}, A)=\bigoplus_{n \in \mathbb{N}} \mathcal{O}(n+1) \otimes_{\Sigma_{n}} \Gamma\left(A^{\otimes n}\right) .
$$

For every $m_{1}, \ldots, m_{n} \in \mathbb{N}$ the structure of an $\mathcal{O}$-algebra on $A$ gives a map

$$
\mathcal{O}\left(m_{1}\right) \otimes \ldots \otimes \mathcal{O}\left(m_{n}\right) \otimes A^{\otimes m} \rightarrow A^{\otimes n}
$$

where $m=m_{1}+\ldots+m_{n}$. Applying the functor $\Gamma$ we obtain a collection of maps

$$
\mu_{m_{1}, \ldots, m_{n}}: \mathcal{O}\left(m_{1}\right) \otimes \ldots \otimes \mathcal{O}\left(m_{n}\right) \otimes \Gamma\left(A^{\otimes m}\right) \rightarrow \Gamma\left(A^{\otimes n}\right)
$$


CYCLIC OPERADS AND CHORD DIAGRAMS

and the following (non-commutative!) diagram

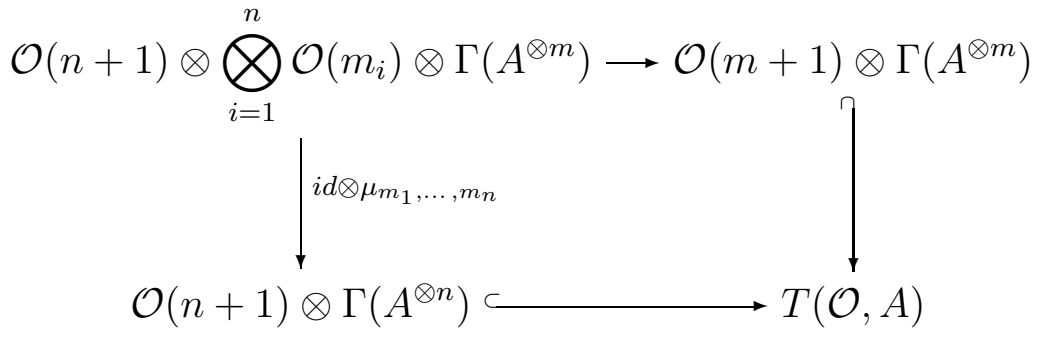

5.1.3. Definition. The external enveloping algebra $U(\mathcal{O}, A)$ is the quotient of the external tensor algebra (38) modulo the weakest equivalence relation making the above diagrams commutative for all $n$, $m_{1}, \ldots, m_{n}$.

Unlike $\mathfrak{U}(\mathcal{O}, A)$ which is defined only when the internal enveloping $\mathcal{U}(\mathcal{O}, A)$ exists, the external enveloping algebra $U(\mathcal{O}, A)$ is defined for all $\mathcal{O}, \mathcal{C}$, and $A$.

5.1.4. The enveloping algebras $\mathfrak{U}(\mathcal{O}, A)$ and $U(\mathcal{O}, A)$ are connected as follows.

Let $A$ be an $\mathcal{O}$-algebra in a tensor category $\mathcal{C}$. For $n=1,2, \ldots$ consider a map

$$
\mathcal{O}(n+1) \otimes_{\Sigma_{n}} \Gamma\left(A^{\otimes n}\right) \rightarrow \mathfrak{U}(\mathcal{O}, A)
$$

defined as the composition

$$
\begin{aligned}
\mathcal{O}(n+1) \otimes_{\Sigma_{n}} \Gamma\left(A^{\otimes n}\right) & \rightarrow \Gamma\left(\mathcal{O}(n+1) \otimes_{\Sigma_{n}} A^{\otimes n}\right) \\
& \rightarrow \Gamma(\mathcal{T}(\mathcal{O}, A)) \rightarrow \Gamma(\mathcal{U}(\mathcal{O}, A))=\mathfrak{U}(\mathcal{O}, A)
\end{aligned}
$$

These maps are compatible with the structure maps defining operations in $\mathcal{O}$ and $A$ and therefore we have a canonical homomorphism

$$
\epsilon_{A}: U(\mathcal{O}, A) \rightarrow \mathfrak{U}(\mathcal{O}, A)
$$

5.1.5. Both external enveloping algebras $\mathfrak{U}(\mathcal{O}, A)$ and $U(\mathcal{O}, A)$ act functorially on all $(\mathcal{O}, A)$-modules.

Let $M$ be an $(\mathcal{O}, A)$-module. For an element $u \in U(\mathcal{O}, A)$ choose a representative $\tilde{u} \in \mathcal{O}(n+1) \otimes \Gamma\left(A^{\otimes n}\right)$ and define an endomorphism $\rho$ of $M$ as the composition

$$
M=\mathbb{I} \otimes M \stackrel{\tilde{u} \otimes \operatorname{id}_{M}}{\longrightarrow} \mathcal{O}(n+1) \otimes A^{\otimes n} \otimes M \rightarrow M,
$$

where the second map is given by the $(\mathcal{O}, A)$-module structure on $M$. A straightforward check shows that $\rho$ does not depend on the choice of the representative $\tilde{u}$ and defines a ring homomorphism

$$
\rho: U(\mathcal{O}, A) \rightarrow \operatorname{End}(M) \text {. }
$$


If the internal enveloping algebra $\mathcal{U}(\mathcal{O}, A)$ exists, one can similarly construct a canonical homomorphism

$$
\mathfrak{U}(\mathcal{O}, A) \rightarrow \operatorname{End}(M)
$$

In this case the homomorphism $\rho$ (41) coincides with the composition of homomorphisms (42) and $\epsilon_{A}$.

5.1.6. Let $i: \mathcal{O} \rightarrow \mathcal{P}$ be a map from an operad $\mathcal{O}$ to a PROP $\mathcal{P}$. The object $\mathbf{1} \in \mathcal{P}$ has a natural $\mathcal{O}$-algebra structure. We will denote this $\mathcal{O}$-algebra by $\mathbb{L}$. Its external enveloping algebra $U(\mathcal{O}, \mathbb{L})$ will be denoted by $U(\mathcal{O}, \mathcal{P})$ to stress the role of the PROP $\mathcal{P}$. Let now $\mathcal{C}$ be a tensor category and let $A$ be a $\mathcal{P}$-algebra in $\mathcal{C}$. Then $A$ admits a natural structure of an $\mathcal{O}$-algebra in $\mathcal{C}$ and we have a canonical ring homomorphism

$$
U(\mathcal{O}, \mathcal{P}) \rightarrow U(\mathcal{O}, A)
$$

induced by the compatible collection of vector space homomorphisms

$$
\Gamma(\mathbf{n}) \rightarrow \Gamma\left(A^{\otimes n}\right) .
$$

5.2. Lie algebras. Until the end of this section we assume $\mathcal{O}=$ LIE. In this case we will write $U(\mathfrak{g})$ instead of $U(\mathrm{LIE}, \mathfrak{g})$, and similarly for $\mathfrak{U}(\mathfrak{g})$ and $\mathcal{U}(\mathfrak{g})$.

We are going to apply the above constructions to the PROPs $\mathrm{LIE}^{C}$ and $\operatorname{LIE}^{M}$ responsible for Casimir and metric Lie algebras (see Section 3.1).

\subsubsection{Definition. The algebras}

$$
\mathbb{L}^{M}=\mathbf{1} \in \operatorname{LIE}^{M} \quad \text { and } \quad \mathbb{L}^{C}=\mathbf{1} \in \operatorname{LIE}^{C}
$$

are called respectively the universal metric Lie algebra and universal Casimir Lie algebra. Their external enveloping algebras will be denoted respectively by

$$
U^{M}=U\left(\operatorname{LIE}^{M}\right) \text { and } \quad U^{C}=U\left(\operatorname{LIE}^{C}\right) .
$$

Corollaries 3.6 .7 and 3.6 .8 yield the following result.

5.2.2. Proposition. The isomorphism (28) induces an isomorphism

$$
\operatorname{LIE}^{M}(\mathbf{0}, \mathbf{0}) \otimes U^{C} \stackrel{\sim}{\longrightarrow} U^{M}
$$

In particular, it implies the following 
CYCLIC OPERADS AND CHORD DIAGRAMS

\subsubsection{Corollary. The homomorphism}

$$
i: U^{C} \rightarrow U^{M}
$$

induced by the functor $\mathrm{LIE}^{C} \rightarrow \mathrm{LIE}^{M}(1 \mathrm{~g})$ is injective.

5.2.4. In order to study relations of the universal enveloping algebras $U^{M}$ and $U^{C}$ of the universal metric and Casimir Lie algebras $\mathbb{L}^{M}$ and $\mathbb{L}^{C}$ with knot invariants (see Section 7 ) we would like to have a description of these algebras in terms of the internal enveloping algebras as in Definition 5.1.2. This seems to be impossible since the tensor categories $\mathrm{LIE}^{C}$ and $\mathrm{LIE}^{M}$ do not admit colimits. However, this difficulty can be resolved following an idea of [EK]. Since the construction in [EK] misses some important details, we present a detailed construction of the algebra $\mathcal{U}(\mathbb{L})$ here.

Let $i: \operatorname{LIE} \rightarrow \mathcal{P}$ be a morphism from LIE to a PROP $\mathcal{P}$. Following [EK], we construct an internal enveloping algebra $\mathcal{U}(\mathbb{L})$ in an certain extension of the tensor category $\mathcal{P}$.

Using this construction we prove Theorem 5.2.11 showing that under mild conditions the internal enveloping algebra of a Lie algebra in a tensor category exists and the two versions of an external enveloping algebra are canonically isomorphic.

5.2.5. Karoubi extension. We will use the following version of the Karoubi extension of a linear category (see $\mathbb{M a n}$, Sect. 5).

Let $\mathcal{C}$ be a $k$-linear category with a collection $\mathcal{X}$ of pairs $(X, e)$, where $X \in \operatorname{Ob} \mathcal{C}$ and $e \in \operatorname{Hom}(X, X)$ is an idempotent and for each $X \in \mathrm{Ob} \mathcal{C}$ the pair $(X, 0)$ belongs to $\mathcal{X}$.

Definition. The Karoubi extension of $\mathcal{C}$ with respect to the collection $\mathcal{X}$ is the category $\widetilde{\mathcal{C}}$ whose objects are pairs $(X, e) \in \mathcal{X}$ and morphisms from $\left(X, e_{X}\right)$ to $\left(Y, e_{Y}\right)$ are maps $f \in \operatorname{Hom}_{\mathcal{C}}(X, Y)$, such that

$$
e_{Y} \circ f=0 \text { and } f \circ e_{X}=0 .
$$

Informally speaking, the object $(X, e)$ in the category $\widetilde{\mathcal{C}}$ corresponds to the kernel of $e: X \rightarrow X$.

5.2.6. Category $\widehat{\mathcal{P}}$. The extension $\widehat{\mathcal{P}}$ of the category $\mathcal{P}$ is constructed in two steps.

First, we construct the Karoubi extension $\widetilde{\mathcal{P}}$ of the category $\mathcal{P}$ adding kernels of the idempotents in $k \Sigma_{n} \subseteq \mathcal{P}(n, n), n=1,2, \ldots$ The objects 
of the category $\widetilde{\mathcal{P}}$ correspond to direct summands of regular representations of the symmetric groups $\Sigma_{n}$. Note that if $e_{1}, e_{2}$ are two different idempotents of $k \Sigma_{n}$ corresponding to isomorphic direct summands of the regular representation of $\Sigma_{n}$, then the corresponding objects of $\widetilde{\mathcal{P}}$ are isomorphic.

After that we add to $\widetilde{\mathcal{P}}$ all direct sums of objects so that the irreducible objects are "of finite type". Namely, let

$$
\hat{\Sigma}=\coprod \hat{\Sigma_{n}}
$$

be the set of irreducible representations of the symmetric groups for $n=1,2, \ldots$ Then the isomorphism classes of irreducible objects $[V]$ in $\widehat{\mathcal{P}}$ are numbered by $V \in \widehat{\Sigma}$.

Furthermore, for $X=\oplus_{i \in I}\left[V_{i}\right]$ and $Y=\oplus_{j \in J}\left[V_{j}\right]$, one has

$$
\operatorname{Hom}_{\widehat{\mathcal{P}}}(X, Y)=\prod_{i \in I} \bigoplus_{j \in J} \operatorname{Hom}_{\widetilde{\mathcal{P}}}\left(\left[V_{i}\right],\left[V_{j}\right]\right) .
$$

The tensor category structure on $\mathcal{P}$ can be uniquely extended to $\widehat{\mathcal{P}}$ so that the functor $\otimes$ commutes with coproducts.

5.2.7. To construct the enveloping algebra of $\mathbb{L}$ we use the PoincaréBirkhoff-Witt theorem which allows one to realize it as a symmetric algebra with a deformed multiplication.

Let $\mathcal{L}(V)$ be the free Lie algebra generated by $V \in$ Vect. The symmetrization map

$$
s: \mathcal{S}(\mathcal{L}(V)) \rightarrow \mathcal{U}(\mathcal{L}(V))=\mathcal{T}(V)
$$

from the symmetric algebra of $\mathcal{L}(V)$ to the tensor algebra of $V$ which identifies with the enveloping algebra of $\mathcal{L}(V)$ is bijective by the PoincaréBirkhoff-Witt theorem. This fact can be interpreted as an isomorphism of polynomial functors

$$
s: \mathcal{S} \circ \mathcal{L} \rightarrow \mathcal{T}
$$

which is equivalent to an infinite collection of identities in the representation rings of symmetric groups.

Any polynomial functor on Vect defines a functor on $\widehat{\mathcal{P}}$. Therefore, isomorphism (47) can be considered as an isomorphism of polynomial functors on $\widehat{\mathcal{P}}$. This can be viewed as the Poincaré-Birkhoff-Witt theorem for free Lie algebras in $\widehat{\mathcal{P}}$.

Let

$$
t: \mathcal{T}(\mathbb{L}) \rightarrow \mathcal{S}(\mathcal{L}(\mathbb{L}))
$$

be the inverse of the isomorphism $s$. 
The Lie algebra structure on $\mathbb{L} \in \widehat{\mathcal{P}}$ defines a map $\mathcal{L}(\mathbb{L}) \rightarrow \mathbb{L}$ which induces a map

$$
m: \mathcal{S}(\mathcal{L}(\mathbb{L})) \rightarrow \mathcal{S}(\mathbb{L})
$$

of symmetric algebras.

\subsubsection{Lemma.}

1. The projection

$$
\pi=m \circ t: \mathcal{T}(\mathbb{L}) \rightarrow \mathcal{S}(\mathbb{L}) .
$$

is the coequalizer of the pair

$$
\mathcal{T}(\mathcal{L}(\mathbb{L})) \underset{\pi_{2}}{\stackrel{\pi_{1}}{\Longrightarrow}} \mathcal{T}(\mathbb{L}),
$$

where $\pi_{1}$ and $\pi_{2}$ are obtained by applying the enveloping algebra functor to two maps $\mathcal{L}(\mathcal{L}(\mathbb{L})) \rightarrow \mathcal{L}(\mathbb{L})$ (note that the enveloping algebra functor is defined on free Lie algebras).

2. The map $\pi$ is a coalgebra morphism with respect to Hopf algebra structures on $\mathcal{T}(\mathbb{L})$ and $\mathcal{S}(\mathbb{L})$ for which the sets of primitive elements coincide with $\mathbb{L}$.

3. The restriction of $\pi$ to $\mathcal{T}^{1}(\mathbb{L}) \subset \mathcal{T}(\mathbb{L})$ is the identity map.

Proof.

1. This is an old Quillen's trick — see [Q], Appendix B. Compare the pair (49) with the following pair

$$
\mathcal{S}(\mathcal{L}(\mathcal{L}(\mathbb{L}))) \underset{\pi_{2}}{\stackrel{\pi_{1}}{\longrightarrow}} \mathcal{S}(\mathcal{L}(\mathbb{L})) .
$$

The pair (50) is known to have $\mathcal{S}(\mathbb{L})$ as a coequalizer since it is obtained by applying the symmetric algebra functor to the split sequence

$$
\mathcal{L}^{2}(\mathbb{L}) \underset{\pi_{2}}{\stackrel{\pi_{1}}{\longrightarrow}} \mathcal{L}(\mathbb{L}) \longrightarrow \mathbb{L}
$$

On the other hand, the pairs (50) and (49) are isomorphic by the Poincaré-Birkhoff-Witt theorem for free Lie algebras (47).

2. It is enough to check that the map $s: \mathcal{S}(\mathcal{L}(\mathbb{L})) \rightarrow \mathcal{T}(\mathbb{L})$ is a morphism of coalgebras. This is equivalent to some identities with polynomial functors which can be verified on their values on $V \in$ Vect. They, in turn, hold because the symmetrization map

$$
s: \mathcal{S}(\mathcal{L}(V)) \rightarrow \mathcal{T}(V)
$$

is an isomorphism of coalgebras for $V \in$ Vect.

3. This is obvious. 
5.2.9. Corollary. (Poincaré-Birkhoff-Witt theorem.) The internal enveloping algebra of $\mathbb{L} \in \widehat{\mathcal{P}}$ exists in $\widehat{\mathcal{P}}$. It is canonically identified with the symmetric algebra $\mathcal{S}(\mathbb{L})$ with multiplication induced from the multiplication in $\mathcal{T}(\mathbb{L})$ via the projection $(48)$.

5.2.10. Corollary. The internal enveloping algebra $\mathcal{U}(\mathbb{L})$ is a Hopf algebra in $\widehat{\mathcal{P}}$. The composition

$$
\mathcal{S}(\mathbb{L}) \stackrel{s}{\longrightarrow} \mathcal{T}(\mathbb{L}) \rightarrow \mathcal{U}(\mathbb{L})
$$

defines the symmetrization map which is an isomorphism of coalgebras.

The construction of the internal enveloping algebra of $\mathbb{L} \in \widehat{\mathcal{P}}$ can be easily generalized.

5.2.11. Theorem. Let $\mathfrak{g}$ be a Lie algebra in a tensor category $\mathcal{C}$. Suppose that $\mathcal{C}$ admits infinite direct sums and that the symmetric powers $\mathcal{S}^{n}(\mathfrak{g})$ exist in $\mathcal{C}$. Then the internal enveloping algebra $\mathcal{U}(\mathfrak{g})$ exists in $\mathcal{C}$. Moreover, if the functor $\Gamma$ commutes with infinite direct sums, the homomorphism (49)

$$
\epsilon_{\mathfrak{g}}: U(\mathfrak{g}) \rightarrow \mathfrak{U}(\mathfrak{g})
$$

is an isomorphism.

Proof. Let $\mathcal{P}=\mathrm{P}(\mathrm{LIE})$ be the PROP (四) generated by operad LIE and let $\mathbb{L} \in \mathcal{P}$ be the universal Lie algebra. According to Corollary 5.2.9, its internal enveloping algebra $\mathcal{U}(\mathbb{L})$ exists in category $\widehat{\mathcal{P}}$.

Let $\widetilde{\mathcal{C}}$ be the Karoubi extension of $\mathcal{C}$ obtained by adding kernels of all idempotents in $\mathcal{C}$. Since $\mathcal{C}$ admits infinite direct sums, $\widetilde{\mathcal{C}}$ admits infinite direct sums as well. Therefore, the functor defining the Lie algebra $\mathfrak{g}$ in $\mathcal{C}$ extends to a functor

$$
\hat{\mathfrak{g}}: \widehat{\mathcal{P}} \rightarrow \widetilde{\mathcal{C}}
$$

Since $\mathcal{U}(\mathbb{L})$ is defined in $\widehat{\mathcal{P}}$ by means of split coequalizers, the image $\hat{\mathfrak{g}}(\mathcal{U}(\mathbb{L}))$ represents an internal enveloping algebra for $\mathfrak{g}$ in $\widetilde{\mathcal{C}}$. Since $\mathcal{C}$ is a full subcategory in $\widetilde{\mathcal{C}}$ and $\hat{\mathfrak{g}}(\mathcal{U}(\mathbb{L}))$ is isomorphic to the symmetric algebra of $\mathfrak{g}$ in $\mathcal{C}$, this proves the existence of the enveloping algebra $\mathcal{U}(\mathfrak{g})$. 
The algebra $\mathcal{U}(\mathfrak{g})$ is a split coequalizer of the pair

$$
\mathcal{T}(\mathcal{L}(\mathfrak{g})) \underset{\pi_{2}}{\stackrel{\pi_{1}}{\longrightarrow}} \mathcal{T}(\mathfrak{g})
$$

constructed similarly to (49). Let us calculate the algebra $\mathfrak{U}(\mathfrak{g})$.

Applying the functor $\Gamma$ to the diagram (52) we get

$$
\Gamma(\mathcal{T}(\mathcal{L}(\mathfrak{g}))) \underset{\pi_{2}}{\stackrel{\pi_{1}}{\longrightarrow}} \Gamma(\mathcal{T}(\mathfrak{g})) .
$$

The coequalizer of this pair can be identified with $U(\mathfrak{g})$. This proves the second part of the theorem.

The rest of this section is devoted to the description of Hopf algebra structures on $U^{C}$ and $U^{M}$ and to proving their commutativity.

\subsection{Hopf algebra structure.}

An internal enveloping algebra $\mathcal{U}(\mathbb{L})$ admits a natural Hopf algebra structure. This does not induce automatically a Hopf algebra structure on $U(\mathcal{P})$ because the map

$$
\operatorname{Hom}(\mathbf{0}, \mathbf{m}) \otimes \operatorname{Hom}(\mathbf{0}, \mathbf{n}) \rightarrow \operatorname{Hom}(\mathbf{0}, \mathbf{m} \otimes \mathbf{n})
$$

is far from being bijective. However, in the two important cases when $\mathcal{P}=\operatorname{LIE}^{C}$ or $\mathcal{P}=\operatorname{LIE}^{M}$ one can define a Hopf algebra structure on $U(\mathcal{P})$.

We return to the use of "the coordinate-free language" of Section 3.2 so that the arguments of our PROPs are finite sets and not natural numbers.

Let $\mathcal{P}=\mathrm{LIE}^{C}$ or $\mathrm{LIE}^{M}$. For each pair $(X, Y)$ of sets we will construct a map

$$
\delta_{X Y}: \mathcal{P}(\emptyset, X \sqcup Y) \rightarrow \mathcal{P}(\emptyset, X) \otimes \mathcal{P}(\emptyset, Y)
$$

co-associative in a natural sense.

5.3.1. Case $\mathcal{P}=\mathrm{LIE}^{C}$.

According to Theorem 3.6.4, we have $\mathcal{P}=\mathbb{P}(\mathcal{M})$, where $\mathcal{M}=$ $\mathbb{M}(\mathrm{LIE})$. Thus,

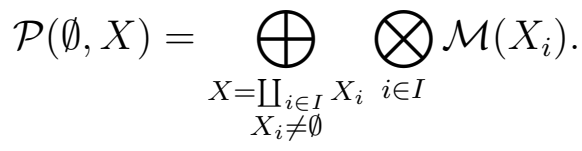

Therefore, the left-hand side of (54) is the direct sum over all partitions of $X \sqcup Y$ whereas the right-hand side is the sum over all decomposable partitions (a union of a partition of $X$ and a partition of $Y$ ). The map $\delta_{X Y}$ can now be defined as a natural projection. 


\subsubsection{Case $\mathcal{P}=\mathrm{LIE}^{M}$.}

According to Theorem 3.6.6, we have $\mathcal{P}=\mathbb{P}_{+}(\mathcal{M})$, where $\mathcal{M}=$ $\mathbb{M}_{+}$(LIE). Thus,

$$
\mathcal{P}(\emptyset, X)=S(\mathcal{M}(\emptyset)) \otimes \bigoplus_{\substack{X=\coprod_{i \in I} X_{i} \neq \emptyset \\ X_{i} \neq \emptyset}} \bigotimes_{i \in I} \mathcal{M}\left(X_{i}\right)
$$

and the map $\delta_{X Y}$ for $\mathcal{P}=\operatorname{LIE}^{M}$ can be defined as the product of the comultiplication

$$
S(\mathcal{M}(\emptyset)) \rightarrow S(\mathcal{M}(\emptyset)) \otimes S(\mathcal{M}(\emptyset))
$$

in the symmetric algebra and the map constructed in 5.3.1

5.3.3. In the cases when $\mathcal{P}$ is either $\mathrm{LIE}^{C}$ or $\mathrm{LIE}^{M}$, one finally defines the comultiplication

$$
\operatorname{Hom}(\mathbf{0}, \mathcal{U}(\mathbb{L})) \rightarrow \operatorname{Hom}(\mathbf{0}, \mathcal{U}(\mathbb{L})) \otimes \operatorname{Hom}(\mathbf{0}, \mathcal{U}(\mathbb{L}))
$$

on $U(\mathcal{P})$ as the composition of

$$
\operatorname{Hom}(\mathbf{0}, \mathcal{U}(\mathbb{L})) \rightarrow \operatorname{Hom}(\mathbf{0}, \mathcal{U}(\mathbb{L}) \otimes \mathcal{U}(\mathbb{L}))
$$

and

$$
\operatorname{Hom}(\mathbf{0}, \mathcal{U}(\mathbb{L}) \otimes \mathcal{U}(\mathbb{L})) \rightarrow \operatorname{Hom}(\mathbf{0}, \mathcal{U}(\mathbb{L})) \otimes \operatorname{Hom}(\mathbf{0}, \mathcal{U}(\mathbb{L}))
$$

where the second map is uniquely defined by (54).

5.3.4. In order to check that the comultiplication (55) defines a Hopf algebra structure on $U(\mathcal{P})$, we have to verify that it is an algebra homomorphism. This is guaranteed by the following Cake lemma claiming that two different ways of cutting a cake $X \cup Y \cup Z \cup T$ into four pieces give the same result.

5.3.5. Lemma. Let $\mathcal{P}$ be $\mathrm{LIE}^{C}$ or $\operatorname{LIE}^{M}$ and let

$$
\gamma_{X, Y}: \Gamma(X) \otimes \Gamma(Y) \rightarrow \Gamma(X \otimes Y)
$$


be the natural morphism. The following diagram

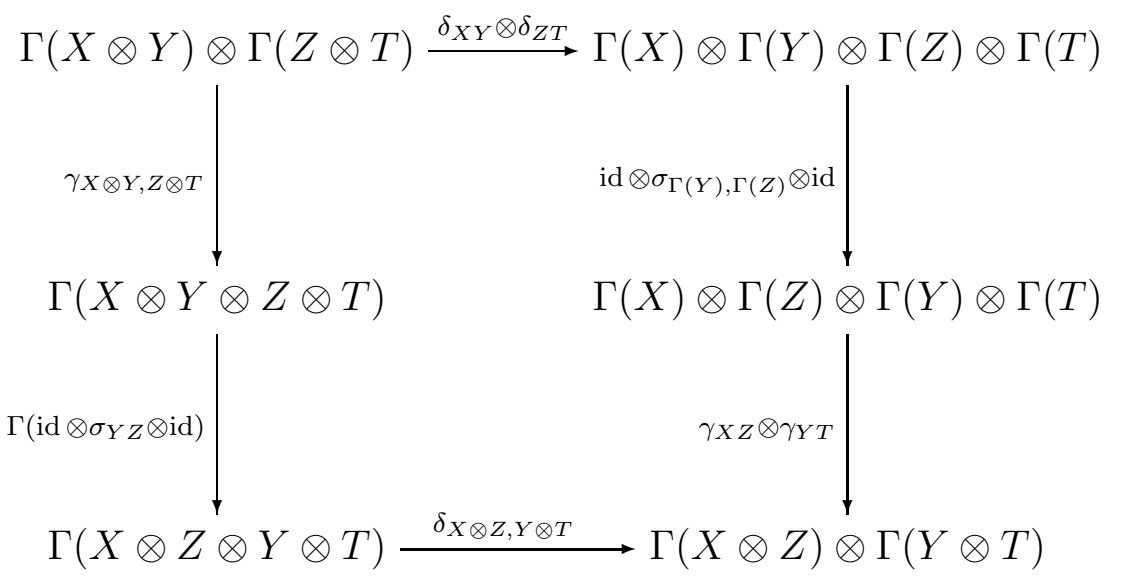

is commutative for each $X, Y, Z, T \in \mathcal{P}$. Here $\sigma$ denotes the commutativity constraint.

5.3.6. It is worthwhile to give the following description of the sets of primitive elements in the algebras $U^{C}$ and $U^{M}$.

Let $\mathcal{P}$ be $\operatorname{LIE}^{C}$ or $\operatorname{LIE}^{M}$ and let $\mathcal{N}$ be $\mathbb{M}(\mathrm{LIE})$ if $\mathcal{P}=\mathrm{LIE}^{C}$ and $\mathbb{M}_{+}(\mathrm{LIE})$ if $\mathcal{P}=\mathrm{LIE}^{M}$.

From the definition of the coproduct in $U(\mathcal{P})$ it follows that the image of the composition

$$
\sum_{n \in \mathbb{N}} \mathcal{N}(n) \rightarrow \sum_{n \in \mathbb{N}} \mathcal{P}(0, n)=\sum_{n \in \mathbb{N}} \operatorname{LIE}(n+1) \otimes_{\Sigma_{n}} \mathcal{P}(0, n) \rightarrow U(\mathcal{P})
$$

consists of primitive elements. The converse is also true.

5.3.7. Proposition. The sets of primitive elements in $U^{C}$ and in $U^{M}$ coincide with the image of (50).

Proof. Enveloping algebras $U^{C}$ and $U^{M}$ are cocommutative Hopf algebras. They are connected since the internal enveloping algebras $\mathcal{U}(\mathbb{L})$ are. They are generated as algebras by the image of (56) which consists of primitive elements. This implies that the image of (56) gives all primitive elements.

\subsection{Centers.}


5.4.1. Definition. Let $A$ be an associative algebra in a tensor category $\mathcal{C}$. An element $z \in \Gamma(A)=\operatorname{Hom}(\mathbb{I}, A)$ is called central in $A$ if the following diagram

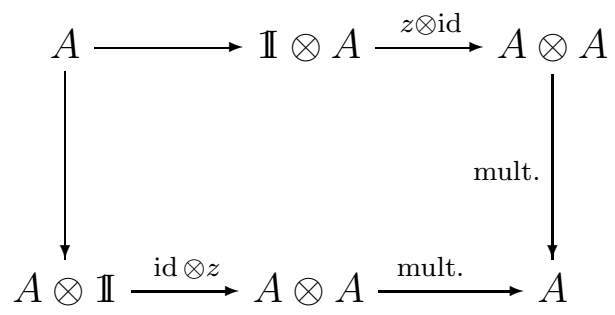

is commutative.

The collection of all central elements of $A$ is called the center of $A$. The center is a commutative subalgebra of $\Gamma(A)$ which we denote $Z(A)$.

5.4.2. Remark. Our notion of a center of an associative algebra in a tensor category is one of several possible.

For example, let $\mathcal{C}$ be the category of super vector spaces. If $A$ is an associative super algebra, $\Gamma(A)$ is its zero component and the center we defined is the degree zero part of (super) center of $A$. It coincides neither with the maximal sub-superalgebra commuting with $A$ nor with the center of $\Gamma(A)$.

5.4.3. Proposition. Let $\mathcal{C}$ be a tensor category admitting infinite direct sums and let $\mathfrak{g} \in \mathcal{C}$ be a Casimir Lie algebra in $\mathcal{C}$, such that the symmetric powers $S^{n}(\mathfrak{g})$ exist. Then the image of the map

$$
U^{C} \rightarrow \Gamma(\mathcal{U}(\mathfrak{g}))=\mathfrak{U}(\mathfrak{g})
$$

induced by the structure tensor functor $\mathfrak{g}: \mathrm{LIE}^{C} \rightarrow \mathcal{C}$ belongs to the center $Z(\mathcal{U}(\mathfrak{g}))$.

Proof. The category $\bmod (\mathfrak{g})$ of representations of $\mathfrak{g}$ is a tensor category with direct sums and $\mathfrak{g}$ endowed with the adjoint action is a Casimir Lie algebra in $\bmod (\mathfrak{g})$. The internal enveloping algebra of $\mathfrak{g}$ in $\bmod (\mathfrak{g})$ is just $\mathcal{U}(\mathfrak{g})$ endowed with the adjoint action of $\mathfrak{g}$. Then the external enveloping algebra is precisely

$$
\operatorname{Hom}_{\bmod (\mathfrak{g})}(\mathbb{I}, \mathcal{U}(\mathfrak{g}))=Z(\mathcal{U}(\mathfrak{g})) .
$$

\section{VASSILIEV INVARIANTS AND LIE ALGEBRAS}

Here we review some facts about Vassiliev knot invariants, the algebra of chord diagrams, and their relationship with Lie algebra-type structures. For more details see [BN, [K1, V]. 
6.1. Singular knots and chord diagrams. A singular knot is an immersion $K: S^{1} \rightarrow \mathbb{R}^{3}$ with a finite number of double self-intersections with distinct tangents. Framed singular knots are defined similarly. Let $\mathcal{K}_{n}$ denote the set of all singular (framed) knots with $n$ double points; in particular, $\mathcal{K}_{0}$ is the set of ordinary (non-singular) knots.

A chord diagram of order $n$ is an oriented circle with $n$ disjoint pairs of points (chords) on it up to an orientation preserving diffeomorphism of the circle. Denote by $\mathcal{D}_{n}$ the set of all chord diagrams with $n$ chords.

Every singular knot $K \in \mathcal{K}_{n}$ has a chord diagram $\operatorname{ch}(K) \in \mathcal{D}_{n}$ whose chords are the inverse images of the double points of $K$.

Every (framed) knot invariant $I$ with values in an abelian group $k$ extends to an invariant of singular knots by the rule

$$
I\left(K_{0}\right)=I\left(K_{+}\right)-I\left(K_{-}\right),
$$

where $K_{0}, K_{+}$, and $K_{-}$are singular knots which differ only inside a small ball as shown on the figure below:
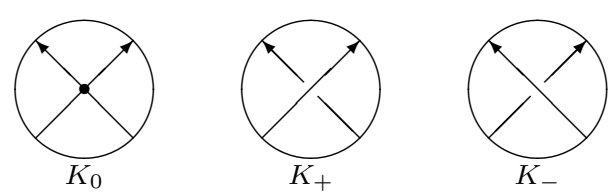

A knot invariant $I$ is called an invariant of order $(\leq) n$ if $I(K)=0$ for any $K \in \mathcal{K}_{n+1}$.

We fix $k$ and denote by $\mathcal{V}_{n}$ the set of all $k$-valued invariants of order $n$. We have an obvious filtration

$$
\mathcal{V}_{0} \subset \mathcal{V}_{1} \subset \mathcal{V}_{2} \ldots \subset \mathcal{V}_{n} \subset \ldots
$$

Elements of

$$
\mathcal{V}=\bigcup_{n} \mathcal{V}_{n}
$$

are called invariants of finite type or Vassiliev invariants.

The definition of Vassiliev invariants implies that the value of an invariant $I \in \mathcal{V}_{n}$ on a singular knot $K$ with $n$ self-intersections depends only on the diagram $c h(K)$ of $K$. In other words, $I$ descends to a function on $\mathcal{D}_{n}$ which we still denote by $I$. These functions satisfy the following relations

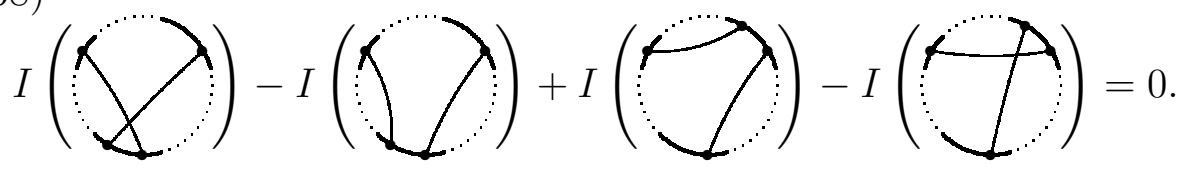


Here each of the four diagrams has $n$ chords, but only chords whose endpoints lie on the solid arcs are shown explicitly. The remaining $n-2$ chords have endpoints on the dotted arcs and are the same in all four diagrams.

A function $W: \mathcal{D}_{n} \rightarrow k$ is called a weight system of order $n$ if it satisfies the four-term relations (58). Denote by $\mathcal{W}_{n}$ the set of all weight systems of order $n$.

Let $\mathcal{A}_{n}$ be the dual space to $\mathcal{W}_{n}$, i.e. the space of formal linear combinations of diagrams from $\mathcal{D}_{n}$ modulo the relations

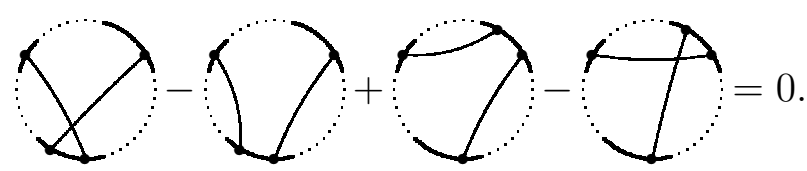

A Vassiliev invariant of order $n$ defines a weight system of order $n$ and it is easy to see that the natural map $\mathcal{V}_{n} / \mathcal{V}_{n-1} \rightarrow \mathcal{W}_{n}$ is injective.

The remarkable fact proved by Kontsevich [K1] is that if $k \supset \mathbb{Q}$ this map is also surjective. In other words, each weight system of order $n$ is a restriction to $\mathcal{D}_{n}$ of some Vassiliev invariant. To prove this Kontsevich constructed a knot invariant

$$
Z: \mathcal{K}_{0} \rightarrow \widehat{\mathcal{A}}
$$

where

$$
\widehat{\mathcal{A}}=\prod_{n \geq 0} \mathcal{A}_{n}
$$

The invariant $Z$ is called Kontsevich's integral.

If $k$ is a commutative ring, then the product of two Vassiliev invariants of orders $m$ and $n$ is a Vassiliev invariant of order $m+n$, therefore $\mathcal{V}$ is a filtered algebra.

The space

$$
\bigoplus_{n \geq 0} \mathcal{W}_{n}=\bigoplus_{n \geq 0} \mathcal{V}_{n+1} / \mathcal{V}_{n}
$$

becomes the associated graded algebra of $\mathcal{V}$ which induces a coproduct $\Delta$ on

$$
\mathcal{A}=\bigoplus_{n \geq 0} \mathcal{A}_{n}
$$

defined as follows.

Let an element $[D] \in \mathcal{A}$ be presented by a chord diagram $D \in \mathcal{D}_{m}$. Then 


$$
\Delta([D])=\sum_{D_{1} \sqcup D_{2}=D}\left[D_{1}\right] \otimes\left[D_{2}\right]
$$

where the sum is taken over all presentations of $D$ as a disjoint union of two subdiagrams $D_{1}$ and $D_{2}$.

The operation of connected sum of diagrams induces on the coalgebra $\mathcal{A}$ a product which makes $\mathcal{A}$ a commutative and cocommutative graded Hopf algebra. It is called the algebra of chord diagrams.

\subsection{Algebra $\mathcal{A}$ and Feynman diagrams.}

There exists an alternative description of the algebra $\mathcal{A}$ of chord diagrams in terms of graphs.

6.2.1. Definition. Feynman diagram of order $p$ is a graph with $2 p$ vertices of degrees 1 or 3 , such that each connected component has at least one vertex of degree 1 and cyclic orderings are fixed on the set of its univalent (external) vertices and on each set of three edges meeting at a trivalent (internal) vertex. Let $\mathcal{F}_{p}$ denote the set of all Feynman diagrams with $2 p$ vertices (up to the natural equivalence of graphs with orientations). The set $\mathcal{D}_{p}$ of chord diagrams with $p$ chords is a subset of $\mathcal{F}_{p}$.

We draw Feynman diagrams by placing their external vertices (legs) on a circle which is oriented counterclockwise.

We assume that the edges meeting at each internal vertex are oriented counterclockwise.

Denote by $\mathcal{G}_{p}$ the vector space generated by Feynman diagrams of order $p$ modulo relations

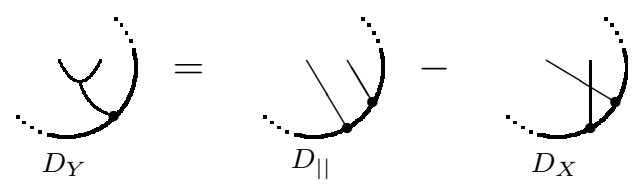

More precisely,

$$
\mathcal{G}_{p}=\left\langle\mathcal{F}_{p}\right\rangle /\left\langle D_{Y}-D_{\|}-D_{X}\right\rangle
$$

where the diagrams $D_{\|}$and $D_{X}$ are obtained from the diagram $D_{Y}$ by replacing its $Y$-fragment by the $\|$ - and $X$ - fragments respectively.

\footnotetext{
${ }^{1}$ Feynman diagrams are called Chinese character diagrams in [BN], but they are indeed Feynman diagrams arising in the perturbative Chern-Simons-Witten quantum field theory.
} 
With this notation we have the following description of the space $\mathcal{A}_{p}$ (see [BN]).

\subsubsection{Proposition.}

(1) The embedding $\mathcal{D}_{p} \hookrightarrow \mathcal{F}_{p}$ induces an isomorphism $\mathcal{G}_{p} \simeq \mathcal{A}_{p}$.

(2) The following local relations hold for internal vertices in Feynman diagrams:

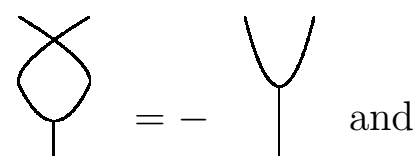

(ii)

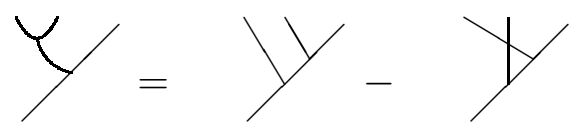

\subsection{Weight systems coming from Lie algebras.}

6.3.1. Here we recall a construction that assigns a family of weight systems to every Lie algebra with an invariant metric.

Let $\mathfrak{g}$ be a Lie algebra in a tensor category $\mathcal{C}$ with a $\mathfrak{g}$-invariant metric $b: \mathfrak{g} \otimes \mathfrak{g} \rightarrow \mathbb{I}$. To each Feynman diagram $F$ with $m$ univalent vertices we assign a tensor

$$
T_{\mathfrak{g}}(F): \mathbb{I} \rightarrow \mathfrak{g}^{\otimes m}
$$

as follows.

The Lie bracket $[]:, \mathfrak{g} \otimes \mathfrak{g} \rightarrow \mathfrak{g}$ can be considered as a tensor

$$
\mathbb{I} \rightarrow \mathfrak{g}^{*} \otimes \mathfrak{g}^{*} \otimes \mathfrak{g} .
$$

The metric $b$ allows us to identify the $\mathfrak{g}$-modules $\mathfrak{g}$ and $\mathfrak{g}^{*}$, and therefore $[$,$] can be considered as a tensor$

$$
f: \mathbb{I} \rightarrow\left(\mathfrak{g}^{*}\right)^{\otimes 3}
$$

and $b$ gives rise to an invariant symmetric tensor

$$
c: \mathbb{I} \rightarrow \mathfrak{g} \otimes \mathfrak{g} .
$$

For a Feynman diagram $F$ denote by $T$ the set of its trivalent vertices, by $U$ the set of its univalent (exterior) vertices, and by $E$ the set of its edges. Taking $|T|$ copies of the tensor $f$ and $|E|$ copies of the tensor $c$ we consider a new tensor

$$
\widetilde{T}_{\mathfrak{g}}(F)=\left(\bigotimes_{v \in T} f_{v}\right) \otimes\left(\bigotimes_{\ell \in E} c_{\ell}\right)
$$

which is considered as a map

$$
\mathbb{I} \rightarrow \mathcal{L}^{F}=\left(\bigotimes_{v \in T}\left(\mathfrak{g}_{v, 1}^{*} \otimes \mathfrak{g}_{v, 2}^{*} \otimes \mathfrak{g}_{v, 3}^{*}\right)\right) \otimes\left(\bigotimes_{\ell \in E}\left(\mathfrak{g}_{\ell, 1} \otimes \mathfrak{g}_{\ell, 2}\right)\right) .
$$


Here $(v, i), i=1,2,3$, mark the three edges meeting at the vertex $v$ (consistently with the cyclic ordering of these edges), and $(\ell, j), j=$ 1,2 , denote the endpoints of the edge $\ell$.

Since $c$ is symmetric and $f$ is completely antisymmetric, the tensor $\widetilde{T}_{\mathfrak{g}}(F)$ does not depend on the choices of the orderings.

If $(v, i)=\ell$ and $(\ell, j)=v$, there is a natural contraction map

$$
\mathfrak{g}_{v, i}^{*} \otimes \mathfrak{g}_{\ell, j} \rightarrow \mathbb{I} .
$$

Composition of all such contractions gives a map

$$
\gamma: \mathcal{L}^{F} \longrightarrow \bigotimes_{u \in U} \mathfrak{g}=\mathfrak{g}^{\otimes m}, \text { where } m=|U| .
$$

The composition of $\gamma$ with $\widetilde{T}_{\mathfrak{g}}(F)$ gives a tensor $T_{\mathfrak{g}}(F): \mathbb{I} \rightarrow \mathfrak{g}^{\otimes m}$.

Often it will be convenient to draw Feynman diagrams with their univalent vertices along a horizontal line.

6.3.2. Example. Let $\mathfrak{g}$ be a metric Lie algebra in Vect with a linear basis $e_{1}, e_{2}, \ldots$ with a metric $b$ given in this basis by $b\left(e_{i}, e_{j}\right)=b_{i j}$. Denote by $\left(b^{i j}\right)$ the inverse of the matrix $\left(b_{i j}\right)$ and by $f_{j k}^{i}$ (or $f_{i j k}$ after lowering indices by means of $b$ ) the structure constants of $\mathfrak{g}$ in the basis $e_{1}, e_{2}, \ldots$.

For the diagrams

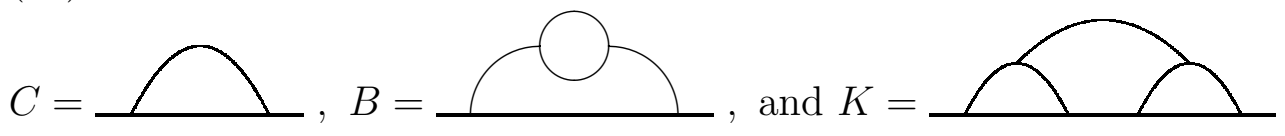

we have

$$
T_{\mathfrak{g}}(C)=\sum_{i j} b^{i j} e_{i} \otimes e_{j}=c,
$$

the Casimir element corresponding to the metric $b$,

$$
T_{\mathfrak{g}}(B)=\sum b^{i s} b^{t j} b^{k p} b^{l q} f_{s k l} f_{p q t} e_{i} \otimes e_{j},
$$

the tensor in $\mathfrak{g} \otimes \mathfrak{g}$ corresponding to the Killing form on $\mathfrak{g}$ under the identification $\mathfrak{g}^{*} \simeq \mathfrak{g}$, and

$$
T_{\mathfrak{g}}(K)=\sum b^{i n} b^{j p} b^{q r} b^{k t} b^{\ell s} f_{n p q} f_{t s r} e_{i} \otimes e_{j} \otimes e_{k} \otimes e_{\ell} .
$$


6.3.3. The tensor $T_{\mathfrak{g}}(F)$ is invariant with respect to the $\mathfrak{g}$-action on $\mathfrak{g}^{\otimes m}$ and its image $W_{\mathfrak{g}}(F)$ in the universal enveloping algebra $U(\mathfrak{g})$ belongs to the center $Z(\mathcal{U}(\mathfrak{g}))=U(\mathfrak{g})^{\mathfrak{g}}$ of the internal enveloping algebra (cf. Section 5.4). The element $W_{\mathfrak{g}}(F) \in Z(\mathcal{U}(\mathfrak{g}))$ does not depend on the place where we cut the circle to obtain a linear ordering of the external vertices of $F$. This gives a well-defined map $\bigoplus_{p}\left\langle\mathcal{F}_{p}\right\rangle \rightarrow Z(\mathcal{U}(\mathfrak{g}))$ which vanishes on the subspace generated by the equations (62) and (61): relations (62) follow from the anticommutativity and the Jacobi identity for the Lie bracket $f$, and (61) in this case is just the definition of the universal enveloping algebra as a quotient of the tensor algebra of $\mathfrak{g}$.

Therefore, for every Lie algebra $\mathfrak{g}$ with an invariant metric we obtain an algebra homomorphism

$$
W_{\mathfrak{g}}: \mathcal{A} \rightarrow Z(\mathcal{U}(\mathfrak{g}))
$$

which is called the universal weight system corresponding to $\mathfrak{g}$. It is universal in the sense that the weight system $W_{\mathfrak{g}, R}$ constructed using a representation $R$ of the Lie algebra $\mathfrak{g}$ (see [BN]) is an evaluation of $W_{\mathfrak{g}}$ :

$$
W_{\mathfrak{g}, R}(D)=\operatorname{Tr}_{R}\left(W_{\mathfrak{g}}(D)\right)
$$

\section{Applications}

\subsection{Algebra of chord diagrams as a universal enveloping al- gebra.}

As an application of the results of Section 3 we describe the algebra $\mathcal{A}$ as the universal enveloping algebra of the universal Casimir Lie algebra and derive some corollaries.

7.1.1. Theorem. The algebra $\mathcal{A}$ of chord diagrams is naturally isomorphic as a Hopf algebra to the external enveloping algebra $U^{C}$ of the universal Casimir Lie algebra $\mathbb{L}^{C}$.

Proof. Let us first construct a homomorphism $a: U^{C} \rightarrow \mathcal{A}$. Define a map $f: \operatorname{LIE}^{C}(0, n) \rightarrow \mathcal{A}$ by assigning to each monomial in variables $c \in \operatorname{LIE}^{C}(0,2)$ and $\lambda \in \operatorname{LIE}^{C}(2,1)$ the element of $\mathcal{A}$ presented by the corresponding Feynman diagram.

The map $f$ extends by linearity to the external tensor algebra (38) and gives an algebra homomorphism

$g: T\left(\operatorname{LIE}, \operatorname{LIE}^{C}\right)=\bigoplus \operatorname{LIE}(n+1) \otimes_{\Sigma_{n}} \operatorname{LIE}^{C}(0, n)=\bigoplus \operatorname{LIE}^{C}(0, n) \rightarrow \mathcal{A}$.

One can easily check that $g$ factors through the projection

$$
T\left(\mathrm{LIE}, \mathrm{LIE}^{C}\right) \rightarrow U^{C}
$$


and gives a well-defined algebra homomorphism $a: U^{C} \rightarrow \mathcal{A}$.

To prove that the map $a$ is also a coalgebra homomorphism we notice that $U^{C}$ is generated as algebra by its primitive elements and therefore it is sufficient to show that a maps primitive elements of $U^{C}$ to primitive elements of $\mathcal{A}$. According to Proposition 5.3.7 the primitive elements of $U^{C}$ are images of $\Gamma(\mathbb{M}(\mathrm{LIE}))$. The map $a$ sends an element of $\Gamma(\mathbb{M}(\mathrm{LIE}))$ to a linear combination of connected graphs which are primitive in $\mathcal{A}$.

Since every Feynman diagram can be presented by a graph whose edges do not have local minima, the map $a$ is onto.

It remains to show that $a$ is injective. Consider the diagram

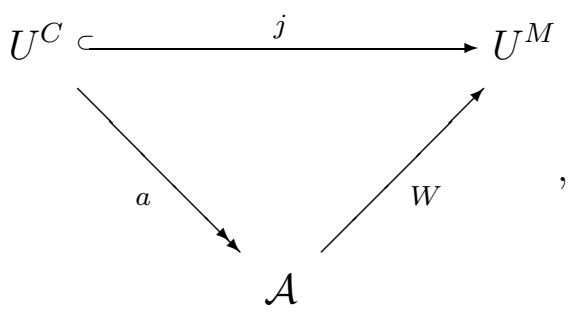

where $W$ is the universal weight system (64) corresponding to the universal metric Lie algebra $\mathbb{L}^{M} \in \mathrm{LIE}^{M}$, and $j$ is the canonical map (46).

Since the diagram is commutative and $j$ is injective by Corollary 5.2.3, this proves the injectivity of $a$.

7.1.2. Denote by $\mathcal{B}$ the symmetric algebra of $\mathbb{L}^{C} \in \operatorname{LIE}^{C}$. It can be described as the algebra generated by graphs analogous to Feynman diagrams except that there is no ordering on the set of its univalent vertices modulo relations (62). (In the terminology of [BN] such graphs are called Chinese characters.)

Theorem 7.1.1 and the Poincaré-Birkhoff-Witt theorem for Lie algebras in tensor categories (see Corollary 5.2.9) give the following result of $[\mathrm{BN}]$.

Theorem. The symmetrization map

$$
\mathcal{B} \stackrel{\sigma}{\longrightarrow} \mathcal{A}
$$

is an isomorphism of vector spaces.

\subsection{Invariants from Casimir Lie algebras.}


7.2.1. Proposition. Let $\mathcal{C}$ be a tensor category with infinite direct sums and $(\mathfrak{g}, t)$ be a Casimir Lie algebra in $\mathcal{C}$ such that the symmetric powers $S^{n}(\mathfrak{g})$ exist in $\mathcal{C}$. There is a canonical algebra homomorphism

$$
\mathcal{A} \rightarrow Z(\mathcal{U}(\mathfrak{g})) .
$$

In particular, any linear functional on the center of $\mathcal{U}(\mathfrak{g})$ gives rise to a sequence of knot invariants.

Proof. It follows from Proposition 5.4.3 and Theorem 7.1.1.

One should not expect this to give new knot invariants by applying traces of finite-dimensional representations of $\mathfrak{g}$ for $\mathcal{C}=$ Vect. However, there might exist nontrivial infinite dimensional Casimir Lie algebras with a known center — this may give something new.

7.2.2. Invariants from metric Lie algebras. Theorem 7.1.1 and Proposition 5.2 .2 imply that any finite type invariant can be obtained from a linear functional on the center of the enveloping algebra of a metric Lie algebra in a tensor $k$-linear category $\mathcal{C}$ (one can take, for instance, $\mathcal{C}=\operatorname{LIE}^{M}$ and $\mathfrak{g}=\mathbb{L}^{M}$ ). This does not contradict the previous claim: it is possible that some invariants coming from a Casimir Lie algebra in the category Vect cannot be obtained from a metric Lie algebra in Vect.

\subsection{Kontsevich integral via Drinfeld's quasi-Hopf algebras.}

Let $(\mathfrak{g}, t)$ be a Casimir Lie algebra in a tensor category $\mathcal{C}$. In [Dr] Drinfeld constructed a ribbon category $\bmod ^{r}(\mathfrak{g})[[h]]$ over $k[[h]]$, where $\bmod ^{r}(\mathfrak{g})$ is the category of rigid $\mathfrak{g}$-modules. Then a version of Reshetikhin's construction $[\mathbb{R}]$ gives a universal knot invariant lying in the center of the category $\bmod ^{r}(\mathfrak{g})[[h]]$.

It is tempting to try to obtain Kontsevich's integral

$$
Z: \mathcal{K}_{0} \rightarrow \prod_{n} \mathcal{A}_{n} h^{n} \subset \mathcal{A}[[h]]
$$

from Theorem 7.1.1 using Drinfeld's construction. We cannot apply Drinfeld's construction directly to $\mathcal{C}=\mathrm{LIE}^{C}$ since this category has no rigid objects. However we can take $\mathcal{C}=\operatorname{LIE}^{M}$ and $\mathfrak{g}=\mathbb{L}^{M}$. We will obtain a knot invariant $D$ with values in the center of $\bmod ^{r}\left(\mathbb{L}^{M}\right)[[h]]$. Let

be the composition

$$
i: U^{C} \rightarrow Z\left(\bmod ^{r}\left(\mathbb{L}^{M}\right)\right)
$$

$$
U^{C} \stackrel{j}{\longrightarrow} U^{M} \rightarrow Z\left(\bmod ^{r}\left(\mathbb{L}^{M}\right)\right) .
$$

For a knot $K$ the value $D(K)$ belongs to the image of $U^{C}[[h]]$ in $Z\left(\bmod ^{r}\left(\mathbb{L}^{M}\right)\right)[[h]]$. If the map $i$ were injective, this would give another 
construction of Kontsevich's invariant $Z$. However, we do not know how to prove the injectivity of $i$.

\subsection{Graph complex.}

We showed above that the functor

$$
\mathbb{M}: \mathrm{CycOp} \rightarrow \mathrm{ModOp}
$$

applied to the cyclic operad LIE naturally leads to the algebra $\mathcal{A}$ of chord diagrams.

In another interesting situation this functor gives Kontsevich's graph complex. We will use the variant of its definition given by Getzler and Kapranov GKM.

Let $\mathcal{O}$ be a cyclic Koszul operad (see [GKC], 3.2). The operad $\mathcal{O}_{\infty}$ responsible for the homotopy $\mathcal{O}$-algebras admits a natural cyclic structure (see GKO, 5.4). For every non-empty finite set $X$ the space $\mathcal{O}_{\infty}(X)$ is a complex whose only cohomology (in degree zero) is canonically isomorphic to $\mathcal{O}(X)$.

The functor $\mathbb{M}$ applied to $\mathcal{O}_{\infty}$ gives a modular operad $\mathbb{M}\left(\mathcal{O}_{\infty}\right)$ in the category of complexes. The quasi-isomorphism $\mathcal{O}_{\infty} \rightarrow \mathcal{O}$ induces a morphism $\mathbb{M}\left(\mathcal{O}_{\infty}\right) \rightarrow \mathbb{M}(\mathcal{O})$.

The operad $\mathcal{O}_{\infty}$ is a free graded cyclic operad generated by $\mathcal{O}^{\perp}$, where $\mathcal{O}^{\perp}$ is the quadratic cooperad dual to $\mathcal{O}$ (see GJ]). One has

$$
\mathcal{O}^{\perp}=\left(\mathcal{O}^{!}\{-1\}\right)^{*},
$$

where $\mathcal{O}$ ! is the operad Koszul dual to $\mathcal{O}$.

According to Remark 3.6.3, the modular operad $\mathbb{M}\left(\mathcal{O}_{\infty}\right)$ considered without the differential is freely generated by $\mathcal{O}^{\perp}=\mathcal{O}\{-1\}^{*}$. Together with the differential, this gives the graph complex corresponding to the cyclic operad $\mathcal{O}$.

The graph complex $\mathbb{M}\left(\mathcal{O}_{\infty}\right)$ can be described in terms of the Feynman transform of [GKM as follows. The cyclic operad structure on $\mathcal{O}^{!}$defines on $\mathcal{O}^{!}\{-1\}$ a structure of an anticyclic operad (see [GKO, 2.11). Therefore by setting

$$
\left(\mathcal{O}^{!}\{-1\}\right)^{n}(X)=0 \text { for } n>0
$$

we can consider $\mathcal{O} !\{-1\}$ as a twisted modular operad with the twist given by the dualizing cocycle (see [GKM], Sect. 4). Then the inverse Feynman transform functor $\mathrm{F}^{-1}$ sends $\mathcal{O} !\{-1\}$ to the modular operad $\mathbb{M}\left(\mathcal{O}_{\infty}\right)$.

7.5. BGRT conjecture. Following the analogy between Lie algebras and the algebras $\mathcal{A}$ and $\mathcal{B}$ of chord diagrams, Bar-Natan, Garoufalidis, 
Rozansky, and Thurston BGRT formulated a conjecture on the explicit form of the algebra isomorphism between certain modifications $\mathcal{A}^{\prime}$ and $\mathcal{B}^{\prime}$ of the algebras $\mathcal{A}$ and $\mathcal{B}$ (see below). I $^{\prime}$

As we saw in 7.1.1, the algebras $\mathcal{A}$ and $\mathcal{B}$ are isomorphic respectively to the universal enveloping algebra (which is commutative in this case) and the symmetric algebra of a certain Lie algebra in a tensor category.

This allows to derive the BGRT conjecture from our Theorem 7.1.1, Proposition 5.2.2, and Kontsevich's theorem on Duflo-Kirillov isomorphism in arbitrary rigid tensor category (see [K2], 8.3).

Denote by $\mathcal{A}^{\prime}$ and $\mathcal{B}^{\prime}$ the spaces generated by trivalent graphs similar to the spaces $\mathcal{A}$ and $\mathcal{B}$ (see Sections 6.2 and 7.1.2) except that connected components with no univalent vertices are allowed. According to Proposition 5.2.2 they can be described in our notation as $\mathcal{A}^{\prime}=U^{M}$ and $\mathcal{B}^{\prime}=\mathcal{S}\left(\mathbb{L}^{M}\right)$, and we obtain the BGRT conjecture.

\subsubsection{Theorem. There is a natural algebra isomorphism}

$$
\mathcal{B}^{\prime} \rightarrow \mathcal{A}^{\prime}
$$

given by the Duflo-Kirillov formula.

\section{REFERENCES}

[A] J.F. Adams, Infinite loop spaces, Annals of Mathematics Studies, 90, 1978.

[BV] J. M. Boardmann, R. M. Vogt, Homotopy invariant algebraic structures on topological spaces, Lecture Notes in Math., 347, 1973.

[D] P. Deligne, Catégories tannakiennes, Grothendieck Festschrift, vol. II, Progr. Math. 87 (1990), 111-195,

[DM] P. Deligne, J. Milne, Tannakian categories, Lecture Notes in math., 900 (1982), 101-228.

[Dr] V. Drinfeld, Quasi-Hopf algebras, Algebra i Analiz, 1 (1989), no. 6, 114148 (English translation: Leningr. Math. J. 1 (1990)).

[GJ] E. Getzler, J. Jones, Operads, homotopy algebra, and iterated integrals for double loop spaces, preprint hep-th/9403055.

[GKC] E. Getzler, M. Kapranov, Cyclic operads and cyclic homology, Geometry, topology, and physics, International Press, Cambridge, MA, 1995, 167201.

[GKM] E. Getzler, M. Kapranov, Modular operads, Compositio Math. 110 (1998), 65-126.

[EK] P. ,Etingof, D. Kazhdan, Quantization of Lie bialgebras, II, Selecta Math., New Ser., 4 (1998), 213-231.

[HS] V. Hinich, V. Schechtman, Homotopy Lie algebras, Adv. Soviet Math., 16, Part 2, (1993) 1-28.

\footnotetext{
${ }^{2}$ According to Bar-Natan, the conjecture is now "multiply proven", see [Mo] and several other txts in preparation.
} 
[M] S. Mac Lane, Categorical algebra, Bull. AMS 71 (1965), 40-106.

[Man] Yu. Manin, Correspondences, motifs and monoidal transformations, (Russian) Mat. Sb. (N.S.) 77 (1968), 475-507.

[May] P. May, The geometry of iterated loop spaces, Lecture Notes in Math. 271 (1972).

[Q] D. Quillen, Rational homotopy theory, Ann. of Math. (2) 90 (1969), 205-295.

[BN] D. Bar-Natan, On the Vassiliev knot invariants, Topology, 34 (1995), 423-472.

[BGRT] D. Bar-Natan, S. Garoufalidis, L. Rozansky, D. Thurston, Wheels, wheeling, and the Kontsevich integral of the unknot, preprint q-alg/9703025.

[Kn] J. Kneissler, The number of primitive Vassiliev invariants up to degree 12, preprint, q-alg/9706022.

[K1] M. Kontsevich, Vassiliev's knot invariants, Adv. Soviet Math., 16, Part 2 (1993), 137-150.

[K2] M. Kontsevich, Deformation quantization of Poisson manifolds, I, preprint q-alg/9709040.

[Mo] T. Mochizuki, On the Kirillov-Duflo type morphism, preprint, 1999.

[R] N. Reshetikhin, Quasitriangular Hopf algebras and invariants of tangles, Algebra i Anal. 1 (1989), no. 2, 169-188 (English translation: Leningr. Math. J. 1 (1990) 491-513).

[V] A. Vaintrob, Vassiliev knot invariants and Lie S-algebras, Math. Res. Lett. 1 (1994), 579-595.

[Vog] P. Vogel, Algebraic structures on modules of diagrams, preprint 1997, Invent. Math., to appear.

Department of Mathematics, University of Haifa, Mount Carmel, HAIFA 31905, ISRAEL

E-mail address: hinich@math.haifa.ac.il

Institut des Hautes Études Scientifiques, Bures-Sur-Yvette, 91440

France; Address after September 1, 2000: Department of Mathematics, University of Oregon, Eugene, OR 97403, USA

E-mail address: vaintrob@math.uoregon.edu 